\title{
Valoración de opciones financieras call en contexto de no normalidad, bajo la aproximación de Edgeworth
}

\author{
Valuation of Call financial options in a context \\ of non-normality: Under the Edgeworth Approach
}

Katty Johanna Acosta-Rueda*

* Magíster en Finanzas, Universidad Externado de Colombia; economista Profesional de la Subgerencia Económica, Transmilenio S.A. [Katty.acosta01@gmail.com], [ORCID: 00000001-5189-2262].

Artículo recibido el 15 de octubre de 2020.

Aceptado el 05 de diciembre de 2020.

Para citar este artículo:

Acosta-Rueda, K. J. (2020). Valoración de opciones financieras call en contexto de no normalidad, bajo la aproximación de Edgeworth. ODEON, 19, 99-152.

DOI: https://doi.org/10.18601/17941113.n19.05 


\section{Resumen}

El modelo de Black-Scholes es el método universal para la valoración de opciones financieras. Sin embargo, este modelo presenta varias deficiencias que hacen que, al contrastar sus resultados con los precios de mercado observados, se evidencie la necesidad de ajustar los métodos de valoración con supuestos menos simplificadores que permitan incluir aspectos observables en la realidad, que favorezcan la derivación del modelo.

Este modelo es un estándar a nivel de las finanzas puesto que es el marco de valoración que se ha trabajado desde hace varias décadas. Sin embargo, en la mayoría de los mercados, por lo general, la distribución de probabilidad de los retornos de los activos objeto de valoración presenta sesgos y asimetría. No obstante, en los modelos clásicos, dados los supuestos restrictivos y simplificadores, solo se tienen en cuenta los momentos estadísticos de primer y segundo orden (media y varianza, respectivamente). Por tanto, si los momentos de orden superior no son considerados, la estimación del valor teórico de una opción sería incompleta.

Por lo anterior, a través de una aproximación de tipo binomial, se propondrá una metodología que permitirá incorporar momentos estadísticos de orden superior (asimetría y curtosis) para proyectar eventuales escenarios futuros en donde el proceso estocástico del subyacente incorpore la incertidumbre, volatilidad y flexibilidad presentes. Esta aproximación es conocida como expansión de Edgeworth, a partir de la cual se obtiene una distribución de probabilidad que incorpora los momentos estadísticos de orden superior.

Palabras clave: expansión de Edgeworth; distribución de normalidad; asimetría; curtosis; rendimientos.

Clasificación JEL: C02, C13, C16, G12.

\section{Abstract}

The Black-Scholes model is the universal method for valuing financial options. However, this model has several deficiencies that, when contrasting its results with the observed market prices, it is evident the need to adjust the valuation methods with less simplifying assumptions that allow us to include observable aspects in reality that favor the derivation of the model.

This model is a standard at the financial level since it is the valuation framework that has been used for several decades. However, in most markets, in general, the probability distribution of the returns of the assets being valued is biases and asymmetry. However, in the classic models given the restrictive and 
simplifying assumptions, only the first and second order statistical moments (mean and variance) are taken into account. Therefore, if higher order moments are not considered, the estimation of the theoretical value of an option would be incomplete.

To this end, a methodology that will allow incorporating higher order statistical moments (asymmetry and kurtosis) to project eventual future scenarios where the stochastic process of the underlying incorporates the present uncertainty, volatility and flexibility will be proposed through a binomial approach. This approximation is known as Edgeworth expansion, from which a probability distribution is obtained that incorporates higher order statistical moments.

Key words: Edgeworth expansion; normality distribution; asymmetry; kurtosis, yields.

JEL classification: $\mathrm{C} 02, \mathrm{C} 13, \mathrm{C} 16, \mathrm{G} 12$.

\section{Introducción}

Las opciones financieras son un contrato que otorga a su propietario el derecho de negociar un activo específico a un precio fijo en cualquier momento o antes de una fecha determinada. El valor que poseen las opciones está determinado por el perfil de riesgo de los individuos y el tipo de distribución que presenta el precio del subyacente (Cox et al., 1979).

En 1973, Black-Scholes determinaron una solución analítica para el precio de una opción europea de compra ( $\mathrm{call}$ ), bajo los supuestos de mercados financieros sin fricciones, tasas de interés para prestar y pedir prestado iguales y constantes entre la fecha de expedición y su vencimiento, el activo subyacente no paga dividendos y su precio sigue una distribución log-normal.

El modelo de Black-Scholes (tiempo continuo) es una de las metodologías más utilizadas para la valoración de opciones europeas a nivel mundial. Sin embargo, este modelo presenta varias deficiencias que hacen que, al contrastar sus resultados con los precios de mercado observados, se evidencie la necesidad de ajustar los métodos de valoración con supuestos menos simplificadores que permitan incluir aspectos observables en la realidad que favorezcan la derivación del modelo.

La distribución de los rendimientos de los activos financieros y reales a menudo no se ajusta al comportamiento normal, toda vez que existen activos que no cumplen con los supuestos requeridos por los modelos clásicos de valoración, 
tales como: i) los mercados financieros son eficientes, ii) no existen comisiones ni gastos de transacción, iii) las tasas de interés se consideran conocidas y constantes durante el periodo de valoración, iv) se asume varianza constante, y v) los retornos están sujetos a una distribución normal y siguen una caminata aleatoria (Black-Scholes, 1973).

Cabe resaltar que, aunque la distribución normal es aceptada como una aproximación de la distribución de los precios de los activos, de alguna manera se torna incompatible ante la evidencia vinculada a la existencia de ciertos hechos estilizados, generando anomalías con los precios de las opciones (Milanesi, 2014).

Realizar valoración de opciones bajo el clásico modelo Black-Scholes produce distorsiones en la estimación realizada puesto que, en el entorno se observa que la volatilidad propia de los rendimientos de los activos subyacentes es mayor que la asumida bajo un mundo de normalidad, es decir, la distribución gaussiana considera muy poco probable la realización de eventos comúnmente observados en la realidad, como por ejemplo, eventos de índole política e inestabilidad macroeconómica de un país, que pueden incrementar los niveles de incertidumbre de una economía.

La prima de una opción, la cual significa determinar el valor del derivado como consecuencia de las expectativas del mercado frente a los cambios en el precio del subyacente, infiere que la volatilidad es un parámetro fundamental toda vez que mide la variabilidad del precio del activo subyacente. Por tanto, asumir que la volatilidad es constante induce a pensar que el modelo no contempla la incertidumbre sobre cambios en los precios y, por tanto, no se tendría en cuenta como una medida para evaluar el riesgo inherente en la opción.

En la mayoría de los mercados, por lo general, la distribución de probabilidad de los activos objeto de valoración presenta sesgos y asimetrías. No obstante, en los modelos clásicos, dados los supuestos restrictivos y simplificadores, únicamente se tienen en cuenta los momentos estadísticos de primer y segundo orden (media y varianza, respectivamente). Por tanto, si los momentos de orden superior no son considerados, la estimación del valor teórico de una opción sería incompleta.

Con el propósito de contribuir a esta temática, y debido a las limitaciones que exponen los supuestos de la distribución normal que llevan a sesgos en las valoraciones de activos financieros y reales a través de la metodología propuesta por Black-Scholes, se aplicará la expansión de Edgeworth a cinco aproximaciones binomiales diferentes caracterizando en cada caso el efecto de la inclusión de estadísticos de alto orden; asimismo, se verificará si existen diferencias en 
el valor de un derivado en relación con el modelo Black-Scholes. Además, para validar su aplicación, se estimarán los parámetros base para la simulación del modelo a fin de calcular el precio de una opción financiera sobre un activo del mercado accionario colombiano.

Para este fin, a través de una aproximación de tipo binomial se propondrá una metodología que permitirá incorporar momentos estadísticos de orden superior (asimetría y curtosis) para proyectar eventuales escenarios futuros en donde el proceso estocástico del subyacente incorpore la incertidumbre, volatilidad y flexibilidad presente. Esta aproximación es conocida como expansión de Edgeworth, a partir de la cual se obtiene una distribución de probabilidad que incorpora los ya mencionados momentos estadísticos de orden superior (Rubinstein, 1998).

El documento se divide en cinco apartados: se inicia con la revisión de la literatura acerca del estudio de modelos que incluyen los momentos de orden superior en sus procesos de valoración, seguida de la metodología estructurada en dos etapas (modelos binomiales y expansión de Edgeworth); posteriormente se hace la aplicación del modelo al mercado accionario colombiano, luego se hace la interpretación de los resultados y, finalmente, se presentan las conclusiones.

\section{Revisión de la literatura}

En la literatura se puede evidenciar una variedad de investigaciones en las cuales se examina el impacto de la distribución del activo subyacente, resumido por sus momentos, en el precio de la opción. Por ejemplo, Jarrow y Rudd (1982) aproximan la distribución del activo subyacente a través de una generalización de la expansión de Edgeworth, cuya diferencia con la empleada por estos autores es que la técnica original usa la normal estándar como la distribución aproximada. En cambio, en este documento se emplea la expansión de Edgeworth generalizada a cualquier distribución de probabilidad, conocida como la "distribución empírica". Bajo este enfoque logran ajustar el modelo Black-Scholes teniendo en cuenta las discrepancias entre los momentos de la distribución log-normal sobre la cual se basa este modelo y la verdadera distribución del precio del activo.

Por su parte, Qumsiyeh (1990) realiza un estudio en el cual obtiene expansiones asintóticas para la estimación de mínimos cuadrados en modelos de regresión lineal múltiple sin asumir errores normales, lo cual evidencia que, bajo condiciones adecuadas y fácilmente verificables, la estimación estandarizada de mínimos cuadrados tiene una densidad y, a su vez, que la expansión de 
Edgeworth utilizada para la densidad es válida. El autor concluye que usando esta expansión se logra obtener un valor aproximado para la distribución de los mínimos cuadrados más favorables que bajo la aproximación normal.

Posteriormente, Rubinstein (1998) desarrolla una técnica simple para valorar opciones europeas y americanas con retorno de los activos subyacentes neutrales al riesgo, que se apartan de la distribución normal en términos de contar con una asimetría diferente de cero y curtosis mayor a tres. En lugar de especificar toda la distribución neutral al riesgo por el rendimiento y la volatilidad (como en el caso Black-Scholes), esta distribución se especifica también por su tercer y cuarto momento central.

Años más tarde, Filho y Rosenfeld (2004) comparan el modelo Edgeworth con el modelo Black-Scholes para opciones de compra del tipo de cambio dólar/ real negociado en el BM\&F brasileño y también opciones de compra del índice S\&P500 negociado en la Bolsa Mercantil de Chicago. Estos autores comparan la sonrisa de volatilidad obtenida en ambos modelos y concluyen que con datos del mercado esta extensión reduce significativamente la sonrisa de volatilidad, lo cual implica que los parámetros son más robustos que en el enfoque tradicional.

También, Del Brio et al. (2008) extienden esta técnica a distribuciones multivariadas centrándose en las especificaciones que garantizan la positividad para obtener una densidad multivariada bien definida. Los autores comparan diferentes distribuciones multivariadas "positivas" con la distribución Normal y la $t$ de Student. Sus resultados muestran que las especificaciones propuestas brindan un rendimiento bastante razonable, además de que esta técnica puede ser adaptada no solo a densidades univariadas, sino también a densidades multivariadas.

Por otra parte, Akita y Wakaki (2010) calculan la expansión de Edgeworth de una prueba estadística cuando algunos de los parámetros son grandes, y simulan la bondad de ajuste de su aproximación. De igual forma, calcularon un límite de error para la expansión de Edgeworth y determinaron que el límite derivado es suficientemente pequeño por lo cual concluyen que es muy práctico emplearlo.

En ese mismo año, Basna (2010) realiza una investigación en la cual analiza la expansión de la serie de Edgeworth para estimar la función de distribución para la media estandarizada de variables aleatorias, independientes e idénticamente distribuidas empleando los modelos de celosía. Este autor descubre una aplicación importante de esta técnica para aquellas compañías desarrolladoras de juegos, la cual puede ser usada a fin de reducir la cantidad de tiempo necesario para satisfacer sus solicitudes antes de aprobar cualquier juego. El autor concluye que la 
estimación obtenida por la aproximación de Edgeworth es mucho mejor que la aproximación normal y más cercana a la función de distribución empírica.

Milanesi (2011), a través de modelos binomiales implícitos, infiere el valor de las probabilidades asociadas a los nodos finales proyectados del subyacente incorporando los momentos estadísticos de orden superior (simetría y curtosis) a diferencia del tradicional modelo binomial. El autor emplea una opción americana sin dividendos y calcula las probabilidades y el árbol binomial implícito, donde se evidencian las diferencias de valor entre ambos métodos (binomial tradicional y binomial implícito). El documento concluye resaltando que el éxito del método dependerá del grado de precisión con que se determina el valor del subyacente en el horizonte temporal y de la disponibilidad de información (precios) para inferir las probabilidades implícitas. Las expectativas de los agentes juegan un rol fundamental en la composición del precio, por tanto, estas no pueden sustraerse del proceso de estimación de valor.

Un año después, Milanesi (2012) aplica esta metodología a un caso de valoración de un contrato de opciones del mercado de capitales argentino. Entre sus conclusiones destaca la importancia de trabajar con modelos de valoración de opciones (financieras y reales) que incorporen los momentos de orden superior, sobre todo en aquellos casos de flujos de fondos esperados con valores positivos vinculados a eventos extremos o poco probables en términos de distribución normal.

Por su parte, Bock (2014) desarrolló una versión ajustada de la expansión de Edgeworth para rejillas binomiales con el fin de obtener las características asintóticas de los diversos precios de opciones en el modelo binomial. El autor observó que la expansión de Edgeworth es válida en condiciones muy generales y puede extenderse a dimensiones más grandes, así como a árboles multinomiales. Asimismo, determinó cómo se pueden obtener expansiones para opciones digitales, estándar y de barrera, y cómo estos resultados se pueden usar para mejorar el comportamiento de convergencia.

De igual forma, Milanesi (2014) aplicó la expansión de Edgeworth en el modelo de Black-Scholes para estimar la volatilidad implícita y el impacto en el precio de la opción de los momentos estadísticos de orden superior sobre contratos de opciones del Grupo Financiero Galicia (GGAL), negociados en la Bolsa de Comercio de Buenos Aires (Argentina). Primero, el autor analiza la distribución de probabilidad de los rendimientos del subyacente, luego desarrolla la expansión de Edgeworth y seguidamente el modelo Black-Scholes con el ajuste de la transformación, para la valoración de opciones financieras 
de compra y venta europeas. Posteriormente, con la ecuación transformada e igualada al precio de mercado, a través de un proceso iterativo, obtuvo los valores implícitos correspondientes a desvío, asimetría, curtosis y tipo sin riesgo. Finalmente, el autor concluye que la expansión de Edgeworth permite corregir las desviaciones respecto del comportamiento normal modificando la distribución de probabilidad, con términos que involucran las anomalías observadas, en este caso, asimetría y curtosis.

Por su parte, Rodríguez (2014) analiza la necesidad de encontrar aproximaciones con fórmula analítica cerrada a sumas de variables aleatorias positivas con colas pesadas. Para esto, utiliza las series de Gram-Charlier y Edgeworth, y realiza experimentos sobre sumas de variables log-normales. En resumen, concluye que las series generalizadas aproximan satisfactoriamente a sumas de variables cuando las colas no son excesivamente pesadas o cuando $N$ es grande. Además, en todos los casos, las aproximaciones dadas por la truncación de la serie de Edgeworth son mejores que las correspondientes a la serie de Gram-Charlier.

La aplicación de esta técnica se evidencia en la valoración de opciones reales, como es el caso de la investigación de Ferreira (2015), quién propuso un modelo de análisis y evaluación de proyectos de inversión aplicados a activos reales sobre empresas de base tecnológica, en el que incorpora momentos estadísticos de orden superior (asimetría y curtosis), que capturan el comportamiento no normal en las variables fundamentales sujetas a análisis, con lo cual concluye que en un contexto con alta volatilidad y con productos que exponen una vida útil muy corta es necesario incorporar el valor de la flexibilidad en el momento de tomar la decisión de aceptar o rechazar un proyecto, para lo cual es muy útil trabajar con distribuciones que no asuman normalidad a fin de gestionar la incertidumbre.

Años más tarde, Brenn et al. (2017) realizan una revisión a las series de Edgeworth y Gram-Charlier como métodos para modelar y aproximar funciones de densidad y presentan una derivación simplificada que destaca tanto las similitudes como las diferencias de las expansiones de la serie y proponen una reformulación de la serie de Edgeworth en términos de los polinomios de Bell, lo cual tiene como resultado una expresión mucho más simplificadora.

Por otra parte, Kao y Xie (2017) elaboraron un estudio en el cual consideraron un spread de opción de tipo europeo y utilizan la expansión de Edgeworth para aproximar el precio de la opción call, observando que cuando se conocen los primeros cuatro momentos, la expansión produce aproximaciones adecuadas para problemas que no tienen soluciones de forma cerrada. Con este artículo 
demuestran que el precio de la opción obtenida por la expansión de Edgeworth proporciona una valiosa información sobre sus constituyentes, es decir, las contribuciones relativas realizadas por los distintos momentos de los precios de los activos, por ejemplo, correlación, asimetría y curtosis.

En años más recientes, Podolskij y Yoshida (2018) realizaron un estudio en el cual presentan nuevos resultados sobre la expansión de Edgeworth para procesos de difusión continua de alta frecuencia, al derivar expansiones asintóticas para funciones del movimiento browniano, y las aplicaron para proporcionar la expansión de Edgeworth de los procesos de difusión.

\section{Metodología}

La valoración de opciones utilizando el modelo Black-Scholes es un estándar a nivel de las finanzas puesto que es el marco de valoración que se ha trabajado desde hace varias décadas. Sin embargo, la valoración por medio de esta metodología tiene varias falencias desde su concepción, toda vez que asume supuestos que no son observados en la realidad (Arnica, 1993).

Las principales falencias corresponden a asumir distribución normal y varianza constante, supuestos que hacen que las valoraciones puedan alejarse de los hechos observados. Como se mencionó en el apartado anterior, autores como Jarrow y Rudd (1982), Rubinstein (1998) y Balieiro y Rosenfeld (2004) proponen ajustar el método de valoración de opciones a través de una técnica que emplea los árboles binomiales incluyendo momentos de orden superior que permitan tener en cuenta las desviaciones presentadas por los precios de las opciones.

Esta técnica propuesta es conocida como expansión de Edgeworth, la cual consiste en aproximar la distribución de los precios de las opciones incluyendo el tercer y cuarto momentos, es decir, asimetría y curtosis, dentro del modelo de valoración. Esta metodología deriva la función de distribución a partir de una distribución aproximada que no está especificada y se caracteriza porque los coeficientes de la expansión son funciones simples de los momentos de la distribución aproximada. Una particularidad de la expansión de Edgeworth es que asume la distribución normal como la distribución aproximada, por tanto, su punto de partida no es una distribución arbitraria, lo cual facilita el desarrollo del método sin perder su esencia (Jarrow y Rudd, 1982).

Ahora bien, como es conocido, el modelo Black-Scholes asume normalidad en la distribución de los rendimientos del activo subyacente, es decir, supone una asimetría igual a cero (0) y una curtosis igual a tres (3); valores distintos a 
estos no se contemplan al momento de valorar opciones a partir de este modelo. Sin embargo, la metodología propuesta por estos autores a través de la serie de Edgeworth, permite cualquier valor de asimetría y curtosis lo cual simplifica la valoración y acerca el resultado obtenido a hechos evidentes en el mercado.

Por tanto, conjugando los árboles binomiales con la aproximación de Edgeworth sobre datos discretos, se logra un ajuste transversal exacto en los precios de las opciones por valorar y se aleja de los supuestos restrictivos del modelo Black-Scholes (Rubinstein, 1998).

Ahora bien, es conocido que la teoría del precio de las opciones no solo ha sido reconocida por su empleo en las finanzas y en la economía a través del modelo de Black-Scholes, sino también por su aplicabilidad por medio de los modelos binomiales gracias a su capacidad de ilustrar las principales ideas de esta teoría y a su flexibilidad analítica que permite obtener el precio de una opción en tiempo discreto bajo los mismos preceptos sobre los cuales se construyó el modelo Black-Scholes.

Con el paso de los años han ido surgiendo diversas variaciones al modelo binomial las cuales, sin perder su esencia, proveen un tratamiento coherente del modelo de valoración de opciones. De hecho, por enunciar algunas versiones del modelo binomial, los modelos de Cox et al. (1979), Rendleman y Bartter (1979) y Jarrow y Rudd (1983) demuestran que los modelos binomiales cuando $N$ suele ser muy grande tienden a converger en el modelo Black-Scholes (Chance, 2007).

Claramente, el modelo binomial no es un modelo único, sino una familia de interpretaciones de un proceso de tiempo discreto que converge en el proceso del movimiento geométrico browniano en el límite continuo, lo que permite obtener el precio de una opción. Por lo anterior, al ser una adaptación al modelo Black-Scholes en tiempo discreto y, por tanto, adoptar sus supuestos de normalidad, seguirá manteniendo supuestos restrictivos que, si bien han sido la base de la teoría de la valoración de opciones financieras, se alejan de los fenómenos observados en la realidad (Chance, 2007).

No obstante, estas falencias que se han evidenciado en los modelos de valoración de opciones, tanto en tiempo discreto como continuo, se pueden ajustar con una leve variación en la estructura de los modelos de tal forma que permitan obtener un precio mucho más ajustado a la realidad y una cobertura más certera para el individuo que quiera acceder a este tipo de contratos financieros. 
En general, la literatura ha evidenciado la existencia de once modelos binomiales $^{1}$, los cuales presentan características diferentes, pero a la vez cada uno proporciona distintas formas de llegar al precio de la opción el cual, indistintamente del modelo empleado, tiende a acercarse al proceso de tiempo continuo (Chance, 2007).

Por lo anterior, y bajo la gran variedad de modelos binomiales existentes y su simplicidad al emplearlos, es posible hacer uso de variaciones que permitan ajustar la distribución del activo subyacente logrando vincular elementos que bajo el precepto del modelo Black-Scholes son excluidos y que pueden lograr robustecer la valoración y diversificar los resultados obtenidos.

Así es como, introduciendo en el precio estimado de la opción los posibles valores que pueden tomar los momentos de orden superior de la función de distribución del activo subyacente es posible llegar a valoraciones más certeras sin alejarse de la simplicidad e intuición que posee el modelo binomial (Milanesi, 2013).

\subsection{Descripción de modelos binomiales}

Los modelos binomiales han atraído mucho la atención en la teoría del precio de las opciones por su capacidad de proporcionar una ilustración clara detrás de la valoración de opciones y, a su vez, por la flexibilidad de las diferentes rutas que puede tomar el precio de una opción, bajo el supuesto de que el precio de un activo subyacente puede cambiar uno a uno dentro de dos posibles resultados, lo cual da un espectro mayor en el campo de la valoración.

Con el paso de los años se ha ido extendiendo a una gran variedad de investigaciones sobre el estudio de los modelos binomiales. No obstante, es bien conocido que el modelo más ampliamente estudiado y reconocido es el propuesto por Cox et al. (1979), pero casi simultáneamente, Rendleman y Bartter (1979) presentaron el mismo modelo de una manera ligeramente diferente (Chance, 2007).

Actualmente, existe una gran variedad de modelos binomiales con muchas similitudes pero, a su vez, con notables diferencias entre ellos. Cada uno desde

1 Según Chance (2007), en la literatura se evidencia la existencia de once modelos binomiales: CRR, RBJRT, Trigeorgis, JKYRB2, Chriss, Wil1, Wil2, JKYABMC2, JKYABMD1, JKYABMD2C y JKYABMD3. 
su enfoque pretende dar un tratamiento coherente al modelo. Sin embargo, algunos de estos enfoques están especificados de forma incorrecta, lo cual permite la existencia de arbitraje y algunos otros modelos no capturan correctamente la volatilidad exógena.

Tres de los principales modelos binomiales existentes, Cox et al. (1979), Rendleman-Bartter (1979) y Jarrow y Rudd (1983) han logrado probar que sus modelos convergen en el modelo Black-Scholes cuando $N$ tiende a infinito. Otros modelos, por su parte, demostraron que la convergencia se puede obtener con menos restricciones en los parámetros (Chance, 2007).

A continuación, se revisarán algunos de los principales modelos binomiales que han surgido en la literatura.

\subsubsection{Cox, Ross y Rubinstein (CRR)}

John Cox et al. (1979) definen el valor de la opción por la ecuación:

$$
\left.C=\left(\pi C_{u}+(1-\pi) C_{d}\right) * e^{-r h}\right)
$$

A partir de la ecuación de probabilidad riesgo neutral definida como:

$$
\pi=\frac{e^{r h}-d}{u-d} ; 1-\pi=\frac{u-e^{r h}}{u-d}
$$

La probabilidad neutral al riesgo es la medida de probabilidad bajo la cual el valor esperado del activo en $t=1$, descontado a $t=0$, es igual al valor del activo en $t=0$, con $r$ como la tasa anual libre de riesgo continuamente compuesta y con $h$ como tamaño del intervalo de tiempo.

CRR luego procede a examinar cómo se comporta su fórmula cuando $N \rightarrow \infty$. Para lo cual eligen $u, d$ y $q$ para que su modelo converja en el límite al valor esperado y la varianza del proceso físico. Adicionalmente, establecen las siguientes condiciones necesarias para el correcto funcionamiento del modelo propuesto:

$$
\begin{aligned}
u d & =1 \\
h^{2} & =0
\end{aligned}
$$


Las anteriores ecuaciones son condiciones necesarias para recuperar la volatilidad correcta, toda vez que la media se obtiene para cualquier $N$.

A partir de lo anterior, se obtienen las ecuaciones para $u$ y $d$ :

$$
\begin{aligned}
& u=e^{\sigma \sqrt{h}} \\
& d=e^{-\sigma \sqrt{h}}
\end{aligned}
$$

Los valores de $u$ y $d$ representan los movimientos que el activo subyacente tendrá hacia arriba $(u)$ y hacia abajo $(d)$ a lo largo del árbol binomial.

Cabe resaltar que CRR reconoce que la única condición necesaria para garantizar ausencia de arbitraje es:

$$
d<1+r<u
$$

Sin embargo, según Chance (2007), las soluciones para $u$ y $d$ están basadas en el proceso continuo y no en el proceso discreto. Por tanto, sus fórmulas para $u$ y $d$ son técnicamente incorrectas para un $N$ finito y no recuperan la correcta volatilidad. No obstante, en el límite sus fórmulas son correctas, toda vez que $u$ y $d$ convergen en 1 y son ínfimamente diferentes de sus valores en el proceso continuo. Además, en el límite recuperan la correcta volatilidad.

\subsubsection{Rendleman y Bartter y Jarrow, Rudd y Turnbull}

Debido a sus similitudes, la discusión del enfoque Rendleman-Bartter (RB) se combina con la discusión del enfoque Jarrow-Rudd (JR) y luego se agrega con el enfoque Jarrow-Turnbull (JT).

Según el enfoque RB, el sistema de ecuaciones se mantiene estable si se cumple que $\mu=r-\frac{\sigma^{2}}{2}$, y las probabilidades neutrales al riesgo tienen valor $\pi^{*}=\frac{1}{2}$; de acuerdo con el enfoque JR se establecen las siguientes fórmulas para $u \mathrm{y} d$ :

$$
u=e^{\mu h+\sigma \sqrt{h}}
$$




$$
d=e^{\mu h-\sigma \sqrt{h}}
$$

Los valores de $u$ y $d$ representan los movimientos que el activo subyacente tendrá hacia arriba $(u)$ y hacia abajo $(d)$ a lo largo del árbol binomial.

La probabilidad neutral al riesgo es la medida de probabilidad bajo la cual el valor esperado del activo en $t=1$, descontado a $t=0$, es igual al valor del activo en $t=0$, con $r$ como la tasa anual libre de riesgo continuamente compuesta y con $h$ como tamaño del intervalo de tiempo.

Años más tarde, JT derivan este modelo, pero hacen una distinción mucho más clara entre el proceso real y el riesgo neutral. Los autores fijan $\pi^{*}$ en su valor libre de arbitraje y muestran que:

$$
\pi^{*}=\frac{e^{r h}-d}{u-d}=\frac{e^{\sigma^{2} h / 2}-e^{-\sigma \sqrt{h}}}{e^{\sigma \sqrt{h}}-e^{-\sigma \sqrt{h}}}
$$

Normalmente, se mostraría que el modelo binomial converge en el modelo BSM utilizando la probabilidad neutral al riesgo. Sin embargo, estos valores para $u$ y $d$ no son consistentes con la ausencia de arbitraje debido a que sus soluciones son obtenidas a partir de la especificación de la media logarítmica. Es decir, son consistentes con la probabilidad neutral al riesgo proxy ${ }^{2} \pi^{*}$. Sin embargo, en el límite se puede demostrar que $\pi$ converge en $\frac{1}{2}$ cuando se usan las fórmulas de JR para $u$ y $d$, con $\pi^{*}=\frac{1}{2}$, con lo cual se obtiene un modelo libre de arbitraje.

Cabe aclarar que la elección de estos autores de $\frac{1}{2}$ como probabilidad, de hecho cualquier probabilidad entre cero y uno, realmente recupera la volatilidad correcta para $N$ finito, pero este resultado solo se obtiene en el proceso real. Una probabilidad neutral al riesgo de $\frac{1}{2}$ solo se obtiene en el límite por el proceso de convergencia. Por tanto, la volatilidad no se recupera para $N$ finito cuando se usa la probabilidad neutral al riesgo, debido a la especificación de $u$ y $d$ empleada en la fórmula.

2 Probabilidad neutral al riesgo obtenida por resolver cualquier condición impuesta. 


\subsubsection{Chriss}

Este modelo especifica la media y la varianza del proceso físico, así:

$$
q u+(1-q) d=e^{\alpha h}
$$

Los valores de $u$ y $d$ representan los movimientos que el activo subyacente tendrá hacia arriba $(u)$ y hacia abajo $(d)$ a lo largo del árbol binomial y la variable $q$ hace referencia a la medida de probabilidad real, obtenida en el mercado.

$\mathrm{Y}$ asume que $q=\frac{1}{2}$ y sus soluciones para $u \mathrm{y} d$ son las siguientes:

$$
\begin{aligned}
& u=\frac{2 e^{\alpha h+2 \sigma \sqrt{h}}}{e^{2 \sigma \sqrt{h}}+1} \\
& d=\frac{2 e^{\alpha h}}{e^{2 \sigma \sqrt{h}}+1}
\end{aligned}
$$

Luego sustituye $r$ por $\alpha$ para obtener los valores neutrales al riesgo, donde $r$ es la tasa anual libre de riesgo continuamente compuesta:

$$
\begin{aligned}
& u=\frac{2 e^{r h+2 \sigma \sqrt{h}}}{e^{2 \sigma \sqrt{h}}+1} \\
& d=\frac{2 e^{r h}}{e^{2 \sigma \sqrt{h}}+1}
\end{aligned}
$$

Debido a que la especificación de la media en este modelo es la media del proceso real, la transformación a neutralidad del riesgo $\alpha=r$ devuelve correctamente la condición de no arbitraje. Por tanto, en el modelo de Chriss $\pi=\pi^{*}=\frac{1}{2}$ para todo $N$; así, el modelo conserva correctamente la condición de no arbitraje y recupera la volatilidad para cualquier número de pasos en el tiempo. 


\subsubsection{Trigeorgis}

Este modelo transforma el proceso original en un proceso logarítmico, es decir $X=\ln S$, y especifica el modelo binomial como el cambio en $X$ o $\Delta X$. Así las cosas, las ecuaciones para el proceso real son:

$$
\begin{gathered}
u=e^{\sqrt{\sigma^{2} h+\mu^{2} h^{2}}} \\
d=e^{-\sqrt{\sigma^{2} h+\mu^{2} h^{2}}} \\
q=\frac{1}{2}+\frac{1}{2} \frac{\mu h}{\sqrt{\sigma^{2} h+\mu^{2} h^{2}}}
\end{gathered}
$$

Si $h^{2}=0$, entonces el modelo Trigeorgis es el mismo que el modelo CRR.

Los valores de $u$ y $d$ representan los movimientos que el activo subyacente tendrá hacia arriba $(u)$ y hacia abajo $(d)$ a lo largo del árbol binomial, la variable $q$ hace referencia a la medida de probabilidad real obtenida en el mercado.

En este modelo se neutraliza el riesgo asumiendo que $\mu=r-\frac{\sigma^{2}}{2}$, y se obtienen las siguientes ecuaciones:

$$
\begin{gathered}
u=e^{\sqrt{\sigma^{2} h+\left(r-\frac{\sigma^{2}}{2}\right)^{2} h^{2}}} \\
d=e^{-\sqrt{\sigma^{2} h+\left(r-\frac{\sigma^{2}}{2}\right)^{2} h^{2}}}
\end{gathered}
$$

En este modelo, la probabilidad neutral al riesgo proviene de la sustitución de $r-\frac{\sigma^{2}}{2}$ por $\mu$ en la fórmula para $q$, donde $r$ es la tasa anual libre de riesgo continuamente compuesta, se obtiene la siguiente ecuación: 


$$
\pi^{*}=\frac{1}{2}+\frac{1}{2} \frac{\left(r-\sigma^{2} / 2\right) h}{\sqrt{\sigma^{2} h+\left(r-\sigma^{2} / 2\right) h^{2}}}
$$

En el límite, $\pi^{*}$ converge en $\frac{1}{2}$ y $\pi$ también converge en $\frac{1}{2}$, por lo que este modelo está libre de arbitraje en el límite.

\subsubsection{Wilmott2}

Este modelo especifica la media y la varianza del proceso real y asume $q=\frac{1}{2}$, donde la variable $q$ es la medida de probabilidad real obtenida en el mercado.

Las soluciones para $u$ y $d$ bajo el proceso de riesgo neutral son las siguientes:

$$
\begin{aligned}
& u=e^{r h}\left(1+\sqrt{e^{\sigma^{2} h}-1}\right) \\
& d=e^{r h}\left(1-\sqrt{e^{\sigma^{2} h}-1}\right)
\end{aligned}
$$

Los valores de $u$ y $d$ representan los movimientos que el activo subyacente tendrá hacia arriba $(u)$ y hacia abajo $(d)$ a lo largo del árbol binomial.

En este modelo se asume $\pi=\frac{1}{2}$ y esta especificación evita correctamente el arbitraje y se recupera la volatilidad en un $N$ finito. Donde $\pi$ es la probabilidad neutral al riesgo y es la medida de probabilidad bajo la cual el valor esperado del activo en $t=1$, descontado a $t=0$, es igual al valor del activo en $t=0$, con $r$ como la tasa anual libre de riesgo continuamente compuesta y con $h$ como tamaño del intervalo de tiempo.

\subsection{Expansión de Edgeworth}

Esta técnica, que tiene sus inicios en los estudios realizados por Schleher (1977) y su posterior desarrollo llevado a cabo por Jarrow y Rudd (1982), tiene como 
finalidad aproximar una distribución de probabilidad dada $F(s)$, conocida como la distribución real, con una distribución aproximada $A(s)$. Esta técnica utiliza la distribución normal como la distribución aproximada.

El primer momento de la distribución aproximada se establece igual al primer momento de la distribución real. La diferencia entre ambas distribuciones se puede expresar como una expansión en serie que involucra las cumulantes de orden superior de ambas distribuciones y sus derivadas.

\subsubsection{Introducción}

Sean $x_{1}, x_{2}, \ldots, x_{n}$ variables aleatorias independientes idénticamente distribuidas, con media $\mu$ y varianza $\sigma^{2}$. Se define la variable aleatoria $X_{n}=-\frac{1}{2} \sum_{j=1}^{n} x_{j}$, entonces por el Teorema del Límite Central, $S_{n}=\frac{X_{n}-\mu}{\sigma} \cdot \sqrt{n}$ se acerca a una distribución normal estándar $N(0,1)$ si $n \rightarrow \infty$; sin embargo, en este caso se estudiará la distribución de probabilidad antes de que $n \rightarrow \infty$ y para esto se deben seguir algunos pasos.

\subsubsection{Expansión de la función característica}

Para simplificar los cálculos, se supondrá en un principio que $\mu=0$ y $\sigma^{2}=1$. De esta forma, se determinará la función característica de $S_{n}$ tomando en cuenta que estará expresada de la siguiente forma:

$$
S_{n}=\frac{X_{n}-\mu}{\sigma} \cdot \sqrt{n}=\sqrt{n} X_{n}=\sqrt{n} \frac{1}{n} \sum_{j=1}^{n} x_{j}=\frac{1}{\sqrt{n}} \sum_{j=1}^{n} x_{j}
$$

Entonces, se define formalmente la función característica como el valor esperado de $e^{i t S_{n}}$ como sigue (Taboga, 2020):

$$
\varphi S_{n}(t)=E\left[e^{i t S_{n}}\right]
$$




$$
\begin{aligned}
& =E\left[e^{i t \frac{1}{\sqrt{n}} \sum_{j=1}^{n} x_{j}}\right] \\
& =E\left[e^{\sum_{j=1}^{n} i \frac{t}{\sqrt{n}} x_{j}}\right] \\
& =E\left[\prod_{j=1}^{n} e^{i \frac{t}{\sqrt{n}} x_{j}}\right]
\end{aligned}
$$

Considerando que $x_{i}$ es independiente, se tiene que:

$$
\varphi S_{n}(t)=\prod_{j=1}^{n} E\left[e^{i \frac{t}{\sqrt{n}} x_{j}}\right]
$$

Donde, por definición, $\varphi x_{i}\left(\frac{t}{\sqrt{n}}\right)=E\left[e^{i \frac{t}{\sqrt{n}} x_{j}}\right]$, así que

$$
\varphi S_{n}(t)=\prod_{j=1}^{n} \varphi x_{i}\left(\frac{t}{\sqrt{n}}\right)
$$

Además, por hipótesis, $x_{i}$ está idénticamente distribuida, por tanto:

$$
\varphi S_{n}(t)=\left[\varphi X \frac{t}{\sqrt{n}}\right]^{n}
$$

\subsubsection{Serie de Taylor (Cañada, 2015)}

Una serie de Taylor es la aproximación de una función mediante una serie de potencias o suma de potencias enteras de polinomios. Formalmente, la serie de Taylor de una función $f(x)$ infinitamente diferenciable en el entorno de un 
número real $a$, es decir, en un intervalo $(a-\varepsilon, a+\varepsilon)(\operatorname{con} \varepsilon>0)$, está definida de la siguiente forma:

$$
f(t)=\frac{f(a)(t-a)^{0}}{0 !}+\frac{f^{\prime}(a)(t-a)^{1}}{1 !}+\frac{f^{\prime \prime}(a)(t-a)^{2}}{2 !}+\ldots
$$

Se debe expandir la función $\varphi X\left(\frac{t}{\sqrt{n}}\right)$, así que se calcula la serie de Taylor de $e^{i \frac{t}{\sqrt{n}} X}$, particularmente la serie de Maclaurin (Serbachi, s. f.), que es el caso en el que $a=0$, como sigue:

$$
\begin{aligned}
& =\frac{e^{i \frac{0}{\sqrt{n}} X}(t-0)^{0}}{0 !}+\frac{e^{i \frac{0}{\sqrt{n}} X}\left(\frac{i X}{\sqrt{n}}\right)(t-0)^{1}}{1 !}+\frac{e^{i \frac{0}{\sqrt{n}} X}\left(\frac{i X}{\sqrt{n}}\right)^{2}(t-0)^{1}}{2 !}+\ldots \\
& =1+\frac{i t X}{\sqrt{n}}+\frac{(i t)^{2} X^{2}}{2 n}+\frac{(i t)^{3} X^{3}}{6 n \sqrt{n}}+\frac{(i t)^{4} X^{4}}{24 n^{2}}+\frac{(i t)^{5} X^{5}}{120 n^{2} \sqrt{n}}+\frac{(i t)^{6} X^{6}}{720 n^{3}}+\ldots
\end{aligned}
$$

Conociendo esta expansión, se puede sustituir en $\varphi X\left(\frac{t}{\sqrt{n}}\right)$, así:

$$
\begin{gathered}
\varphi X\left(\frac{t}{\sqrt{n}}\right)=E\left[e^{i \frac{t}{\sqrt{n}} X}\right] \\
=E\left[1+\frac{i t X}{\sqrt{n}}+\frac{(i t)^{2} X^{2}}{2 n}+\frac{(i t)^{3} X^{3}}{6 n \sqrt{n}}+\frac{(i t)^{4} X^{4}}{24 n^{2}}+\frac{(i t)^{5} X^{5}}{120 n^{2} \sqrt{n}}+\frac{(i t)^{6} X^{6}}{720 n^{3}}+\ldots\right] \\
=E[1]+E\left[\frac{i t X}{\sqrt{n}}\right]+E\left[\frac{(i t)^{2} X^{2}}{2 n}\right]+E\left[\frac{(i t)^{3} X^{3}}{6 n \sqrt{n}}\right]+E\left[\frac{(i t)^{4} X^{4}}{24 n^{2}}\right]+ \\
E\left[\frac{(i t)^{5} X^{5}}{120 n^{2} \sqrt{n}}\right]+E\left[\frac{(i t)^{6} X^{6}}{720 n^{3}}\right]+\ldots
\end{gathered}
$$




$$
\begin{aligned}
= & 1+\frac{i t E[X]}{\sqrt{n}}+\frac{(i t)^{2} E\left[X^{2}\right]}{2 n}+\frac{(i t)^{3} E\left[X^{3}\right]}{6 n \sqrt{n}}+\frac{(i t)^{4} E\left[X^{4}\right]}{24 n^{2}}+ \\
& \frac{(i t)^{5} E\left[X^{5}\right]}{120 n^{2} \sqrt{n}}+\frac{(i t)^{6} E\left[X^{6}\right]}{720 n^{3}}+\ldots
\end{aligned}
$$

Los momentos $E[X]$ y $E\left[X^{2}\right]$ representan la media y la varianza que por hipótesis son 0 y 1 , respectivamente; además, con $i^{2}=-1$, entonces se puede reescribir esta última expresión como:

$$
1-\frac{t^{2}}{2 n}+\frac{(i t)^{3} E\left[X^{3}\right]}{6 n \sqrt{n}}+\frac{(i t)^{4} E\left[X^{4}\right]}{24 n^{2}}+\frac{(i t)^{5} E\left[X^{5}\right]}{120 n^{2} \sqrt{n}}+\frac{(i t)^{6} E\left[X^{6}\right]}{720 n^{3}}+\ldots
$$

A partir del quinto sumando tienen como denominador números mayores que $n^{2}$, por tanto, tienen una cota superior asintótica $\frac{1}{n^{2}}$ cuando $n \rightarrow \infty$, por esta razón se utiliza la notación $O$ Grande ${ }^{3}$. Así,

$$
\varphi X\left(\frac{t}{\sqrt{n}}\right) 1-\frac{t^{2}}{2 n}+\frac{(i t)^{3} E\left[X^{3}\right]}{6 n \sqrt{n}}+\frac{(i t)^{4} E\left[X^{4}\right]}{24 n^{2}}+O\left(\frac{1}{n^{2}}\right)
$$

Es necesario emplear la notación $O$ Grande cuando se trabaja en espacios finitos con la finalidad de indicar a partir de dónde se cumple el teorema. En este caso, hasta el sumando $O\left(\frac{1}{n^{2}}\right)$ se acota la expresión para obtener los términos que permitan dar cumplimiento al teorema que se está desarrollando.

De esta forma, considerando que:

$$
\varphi S_{n}(t)=\left[\varphi X\left(\frac{t}{\sqrt{n}}\right)\right]^{n}
$$


Entonces,

$$
\varphi S_{n}(t)=\left[1-\frac{t^{2}}{2 n}+\frac{(i t)^{3} E\left[X^{3}\right]}{6 n \sqrt{n}}+\frac{(i t)^{4} E\left[X^{4}\right]}{24 n^{2}}+O\left(\frac{1}{n^{2}}\right)\right]^{n}
$$

Por lo que el siguiente paso será desarrollar una técnica que permita expandir esta última expresión, observando que dentro de ella hay tres sumandos de importancia:

$$
1-\frac{t^{2}}{2 n}, \frac{(i t)^{3} E\left[X^{3}\right]}{6 n \sqrt{n}} y \frac{(i t)^{4} E\left[X^{4}\right]}{24 n^{2}}
$$

\subsubsection{Expansión trinomial}

La expansión trinomial (Ahmed, 2006) es una generalización de la expansión binomial y establece que si se consideran tres números reales $a, b, c$, entonces: $(a+b+c)^{n}=\sum_{i+j+k=n}\left(\begin{array}{c}n \\ i, j, k\end{array}\right) a^{i} b^{j} c^{k}$ donde $\left(\begin{array}{c}n \\ i, j, k\end{array}\right)=\frac{n !}{i ! j ! k !}$

Si se considera la suma:

$$
\varphi X\left(\frac{t}{\sqrt{n}}\right)=\underbrace{1-\frac{t^{2}}{2 n}}_{a}+\underbrace{\frac{(i t)^{3} E\left[X^{3}\right]}{6 n \sqrt{n}}}_{b}+\underbrace{\frac{(i t)^{4} E\left[X^{4}\right]}{24 n^{2}}}_{c}+O\left(\frac{1}{n^{2}}\right),
$$

como un trinomio, se puede usar la expansión trinomial para expandir $\left[\varphi X\left(\frac{t}{\sqrt{n}}\right)\right]^{n}$ y tomando en cuenta que la mayoría de los términos tendrán una cota superior asintótica $\frac{1}{n}$ cuando $n \rightarrow \infty$ se consideran los sumandos tales que:

- $\quad i=n, j=0 \mathrm{y} k=0$

- $\quad i=n-1, j=1$ y $k=0$ 
- $i=n-1, j=0$ y $k=1$

- $\quad i=n-2, j=2$ y $k=0$

Así,

$$
\begin{gathered}
{\left[\varphi X\left(\frac{t}{\sqrt{n}}\right)\right]^{n}=\frac{n !}{n !}\left(1-\frac{t^{2}}{2 n}\right)^{n}+\frac{n !}{(n-1) ! 1 !}\left(1-\frac{t^{2}}{2 n}\right)^{n-1}\left(\frac{(i t)^{3} E\left[X^{3}\right]}{6 n \sqrt{n}}\right)} \\
+\frac{n !}{(n-1) ! 1 !}\left(1-\frac{t^{2}}{2 n}\right)^{n-1}\left(\frac{(i t)^{4} E\left[X^{4}\right]}{24 n^{2}}\right) \\
+\frac{n !}{(n-2) ! 2 !}\left(1-\frac{t^{2}}{2 n}\right)^{n-2}\left(\frac{(i t)^{3} E\left[X^{3}\right]}{6 n \sqrt{n}}\right)^{2}+O\left(\frac{1}{n}\right) \\
=\left(1-\frac{t^{2}}{2 n}\right)^{n}+n\left(1-\frac{t^{2}}{2 n}\right)^{n-1}\left(\frac{(i t)^{3} E\left[X^{3}\right]}{6 n \sqrt{n}}+\frac{(i t)^{4} E\left[X^{4}\right]}{24 n^{2}}\right) \\
+\frac{n(n-1)}{2}\left(1-\frac{t^{2}}{2 n}\right)^{n-2}\left(\frac{(i t)^{6} E\left[X^{3}\right]^{2}}{36 n^{2} n}\right)+O\left(\frac{1}{n}\right) \\
=\left(1-\frac{t^{2}}{2 n}\right)^{n}+\left(1-\frac{t^{2}}{2 n}\right)^{n-1}\left(\frac{(i t)^{3} E\left[X^{3}\right]}{6 n \sqrt{n}}+\frac{(i t)^{4} E\left[X^{4}\right]}{24 n^{2}}\right) \\
+\left(1-\frac{t^{2}}{2 n}\right)^{n-2}\left(\frac{(n-1)(i t)^{6} E\left[X^{3}\right]^{2}}{72 n^{2}}\right)+O\left(\frac{1}{n}\right)
\end{gathered}
$$

Para expandir los binomios $\left(1-\frac{t^{2}}{2 n}\right)$ se puede utilizar la expansión binomial, sin embargo, en el numeral 2.2.5., mediante un desarrollo analítico empleando el número $e$, se podrán enlazar estas expresiones con la función exponencial. 


\subsubsection{El número e}

Es el valor límite de la sucesión $\left(1+\frac{a}{n}\right)^{n}$, dicho límite se puede denotar con $e^{a}$ cuando $n \rightarrow \infty$. Sin embargo, desde un principio se ha considerado una distribución de probabilidad de valores muy grandes, pero antes de que $n \rightarrow \infty$. Entonces, mediante un extenso desarrollo algebraico fijando un entero no negativo $k$ se puede establecer que (Hunter, 2014):

$$
\left(1+\frac{a}{n}\right)^{n-k}=e^{a}\left(1+\frac{a(1+k)}{2 n}\right)+O\left(\frac{1}{n}\right)
$$

De esta forma, se puede reescribir $\left(1-\frac{t^{2}}{2 n}\right)$ como $\left(1+\frac{\left(-\frac{t^{2}}{2}\right)}{n}\right)$, y al considerar $a=-\frac{t^{2}}{2}$ se aplica la anterior igualdad para obtener que:

$$
\begin{aligned}
\left(1+\frac{\left(-\frac{t^{2}}{2}\right)}{n}\right)^{n} & =e^{-\frac{t^{2}}{2}}\left(1-\frac{\left(-\frac{t^{2}}{2}\right)\left(-\frac{t^{2}}{2}+0\right)}{2 n}\right)+O\left(\frac{1}{n}\right) \\
& =e^{-\frac{t^{2}}{2}}\left(1-\frac{t^{4}}{8 n}\right)+O\left(\frac{1}{n}\right) \\
\left(1+\frac{\left(-\frac{t^{2}}{2}\right)}{n}\right)^{n-1} & =e^{-\frac{t^{2}}{2}}\left(1-\frac{\left(-\frac{t^{2}}{2}\right)\left(-\frac{t^{2}}{2}+1\right)}{2 n}\right)+O\left(\frac{1}{n}\right)
\end{aligned}
$$




$$
\begin{gathered}
=e^{-\frac{t^{2}}{2}}\left(1-\frac{t^{4}}{8 n}+\frac{t^{2}}{4 n}\right)+O\left(\frac{1}{n}\right) \\
\left(1+\frac{\left(-\frac{t^{2}}{2}\right)}{n}\right)^{n-2}=e^{-\frac{t^{2}}{2}}\left(1-\frac{\left(-\frac{t^{2}}{2}\right)\left(-\frac{t^{2}}{2}+2\right)}{2 n}\right)+O\left(\frac{1}{n}\right) \\
=e^{-\frac{t^{2}}{2}\left(1-\frac{t^{4}}{8 n}+\frac{t^{2}}{4 n}\right)+O\left(\frac{1}{n}\right)}
\end{gathered}
$$

Estas expresiones se pueden sustituir en $\left[\varphi X\left(\frac{t}{\sqrt{n}}\right)\right]^{n}$ para obtener:

$$
\begin{aligned}
& {\left[\varphi X\left(\frac{t}{\sqrt{n}}\right)\right]^{n}=e^{-\frac{t^{2}}{2}}\left(1-\frac{t^{4}}{8 n}\right)} \\
& +e^{-\frac{t^{2}}{2}}\left(1-\frac{t^{4}}{8 n}+\frac{t^{2}}{2 n}\right)\left(\frac{(i t)^{3} E\left[X^{3}\right]}{6 \sqrt{n}}+\frac{(i t)^{4} E\left[X^{4}\right]}{24 n}\right) \\
& +e^{-\frac{t^{2}}{2}}\left(1-\frac{t^{4}}{8 n}+\frac{t^{2}}{2 n}\right)\left(\frac{n(i t)^{6} E\left[X^{3}\right]^{2}}{72 n^{2}}\right) \\
& +e^{-\frac{t^{2}}{2}}\left(1-\frac{t^{4}}{8 n}+\frac{t^{2}}{2 n}\right)\left(\frac{(-1)(i t)^{6} E\left[X^{3}\right]^{2}}{72 n^{2}}\right)+O\left(\frac{1}{n}\right)
\end{aligned}
$$

Al distribuir las sumas con sus factores respectivos, muchos de los sumandos resultantes tendrán una cota superior asintótica $\frac{1}{n}$ cuando $n \rightarrow \infty$, así que $\varphi S_{n}(t)$ será igual a: 


$$
\begin{aligned}
& e^{-\frac{t^{2}}{2}}\left(1-\frac{t^{4}}{8 n}+\frac{(i t)^{3} E\left[X^{3}\right]}{6 \sqrt{n}}+\frac{(i t)^{4} E\left[X^{4}\right]}{24 n}+\frac{(i t)^{6} E\left[X^{3}\right]^{2}}{72 n}\right)+O\left(\frac{1}{n}\right) \\
= & e^{-\frac{t^{2}}{2}}\left(1-\frac{3 t^{4}}{24 n}+\frac{(i t)^{3} E\left[X^{3}\right]}{6 \sqrt{n}}+\frac{(i t)^{4} E\left[X^{4}\right]}{24 n}+\frac{(i t)^{6} E\left[X^{3}\right]^{2}}{72 n}\right)+O\left(\frac{1}{n}\right) \\
= & e^{-\frac{t^{2}}{2}}\left(1+\frac{(i t)^{3} E\left[X^{3}\right]}{6 \sqrt{n}}+\frac{(i t)^{4}\left(E\left[X^{4}\right]-3\right)}{24 n}+\frac{(i t)^{6} E\left[X^{3}\right]^{2}}{72 n}\right)+O\left(\frac{1}{n}\right)
\end{aligned}
$$

\subsubsection{Teorema (la fórmula de inversión) ${ }^{3}$}

Si $X$ es una variable aleatoria que tiene una función de distribución de probabilidad $G_{X}(x)$ y una función característica $\varphi X(t)$ que es integrable, entonces $X$ tiene función de densidad de probabilidad $g_{X}(x)$ que está definida de la siguiente forma (Dávalos, 2012):

$$
g_{X}(x)=G^{\prime}{ }_{X}(x)=\frac{1}{2 \pi} \int_{-\infty}^{\infty} e^{-i t x} \varphi X(t) d t
$$

Se calcula la función de densidad de probabilidad de $S_{n}$ usando la fórmula que provee el teorema. Entonces $g_{S_{n}(x)}$ será igual a:

$$
\frac{1}{2 \pi} \int_{-\infty}^{\infty} e^{-i t x} e^{-\frac{t^{2}}{2}}\left(1+\frac{(i t)^{3} E\left[X^{3}\right]}{6 \sqrt{n}}+\frac{(i t)^{4}\left(E\left[X^{4}\right]-3\right)}{24 n}+\frac{(i t)^{6} E\left[X^{3}\right]^{2}}{72 n}\right) d t
$$

3 Ver Edgeworth expansions en http://personal.psu.edu/drh20/asymp/fall2004/lectures/ edgeworth.pdf 


$$
\begin{aligned}
& =\frac{1}{2 \pi} \int_{-\infty}^{\infty} e^{-i t x} e^{-\frac{t^{2}}{2}} d t+\frac{1}{2 \pi} \int_{-\infty}^{\infty} e^{-i t x} e^{-\frac{t^{2}}{2}} \frac{(i t)^{3} E\left[X^{3}\right]}{6 \sqrt{n}} d t \\
& +\frac{1}{2 \pi} \int_{-\infty}^{\infty} e^{-i t x} e^{-\frac{t^{2}}{2}} \frac{(i t)^{4}\left(E\left[X^{4}\right]-3\right)}{24 n} d t \\
& +\frac{1}{2 \pi} \int_{-\infty}^{\infty} e^{-i t x} e^{-\frac{t^{2}}{2}} \frac{(i t)^{6} E\left[X^{3}\right]^{2}}{72 n} d t \\
& =\frac{1}{2 \pi} \int_{-\infty}^{\infty} e^{-i t x} e^{-\frac{t^{2}}{2}} d t+\frac{E\left[X^{3}\right]}{6 \sqrt{n}} \frac{1}{2 \pi} \int_{-\infty}^{\infty} e^{-i t x} e^{-\frac{t^{2}}{2}}(i t)^{3} d t \\
& +\frac{\left(E\left[X^{4}\right]-3\right)}{24 n} \frac{1}{2 \pi} \int_{-\infty}^{\infty} e^{-i t x} e^{-\frac{t^{2}}{2}}(i t)^{4} d t \\
& +\frac{E\left[X^{3}\right]^{2}}{72 n} \frac{1}{2 \pi} \int_{-\infty}^{\infty} e^{-i t x} e^{-\frac{t^{2}}{2}}(i t)^{6} d t
\end{aligned}
$$

Cada una de las integrales planteadas en esta última igualdad tienen la forma:

$$
\int_{-\infty}^{\infty} e^{-i t x} e^{-\frac{t^{2}}{2}}(i t)^{k} d t
$$

Para facilitar los cálculos posteriores, en el numeral 2.2.7 se plantearán expresiones equivalentes para cada una de las integrales obtenidas en esta sección.

\subsubsection{Los polinomios de Hermite}

Si $\varnothing(x)$ denota la función de densidad de probabilidad de la distribución normal estándar, entonces los polinomios de Hermite $H_{k}(x)$ de orden $k$ se definen como aquellos que satisfacen la siguiente ecuación:

$$
(-1)^{k} \frac{d^{k}}{d x^{k}} \varnothing(x)=H_{k}(x) \varnothing(x)
$$


Los cuales, después de un simple despeje, se pueden calcular de la siguiente forma:

$$
H_{k}(x)=(-1)^{k} \frac{d^{k}}{d x^{k}} \emptyset(x) \frac{1}{\emptyset(x)}
$$

Una vez definidos los polinomios de Hermite se considerará una serie de expresiones que permitirán establecer una relación entre ellos y la fórmula de inversión.

Particularmente, si se aplica la integral que se usa en la fórmula de inversión a la expresión $e^{-\frac{t^{2}}{2}}(i t)^{k}$ se puede obtener que:

$$
\frac{1}{2 \pi} \int_{-\infty}^{\infty} e^{-i t x} e^{-\frac{t^{2}}{2}}(i t)^{k} d t=\frac{1}{2 \pi} \int_{-\infty}^{\infty} \frac{d^{k}}{d x^{k}}\left(e^{-i t x}\right)(-1)^{k} e^{-\frac{t^{2}}{2}} d t
$$

Esto se debe a que para todo entero positivo $k, \frac{d^{k}}{d x^{k}}\left(e^{-i t x}\right)=e^{-i t x}(i t)^{k}(-1)^{k}$, y como se está derivando esta expresión respecto a $x$, se puede incluir toda la integral dentro de la derivada,

$$
(-1)^{k} \frac{d^{k}}{d x^{k}}\left(\frac{1}{2 \pi} \int_{-\infty}^{\infty} e^{-i t x} e^{-\frac{t^{2}}{2}} d t\right)
$$

Justamente, el elemento que se encuentra dentro de la derivada se puede reescribir usando la fórmula de inversión para la función de densidad de probabilidad de la distribución normal estándar, así:

$$
(-1)^{k} \frac{d^{k}}{d x^{k}} \underbrace{\left(\frac{1}{2 \pi} \int_{-\infty}^{\infty} e^{-i t x} e^{-\frac{t^{2}}{2}} d t\right)}_{\varnothing(x)}=(-1)^{k} \frac{d^{k}}{d x^{k}} \emptyset(x)=H_{k}(x) \emptyset(x)
$$

Por tanto, se concluye que:

$$
\frac{1}{2 \pi} \int_{-\infty}^{\infty} e^{-i t x} e^{-\frac{t^{2}}{2}}(i t)^{k} d t=H_{k}(x) \emptyset(x)
$$


Adicionalmente, los polinomios de Hermite se pueden calcular considerando que $\emptyset(x)=e^{-\frac{t^{2}}{2}} \mathrm{y}$ usando la fórmula que se ha planteado para calcularlos.

- Orden 1:

$$
\begin{gathered}
H_{1}(x)=(-1)^{1} \frac{d}{d x}\left(e^{-\frac{x^{2}}{2}}\right) e^{\frac{x^{2}}{2}} \\
H_{1}(x)=(-1)(-x) e^{-\frac{x^{2}}{2}} e^{\frac{x^{2}}{2}} \\
H_{1}(x)=x
\end{gathered}
$$

- Orden 2:

$$
\begin{gathered}
H_{2}(x)=(-1)^{2} \frac{d^{2}}{d x^{2}}\left(e^{-\frac{x^{2}}{2}}\right) e^{\frac{x^{2}}{2}} \\
H_{2}(x)=(1)\left(x^{2}-1\right) e^{-\frac{x^{2}}{2}} e^{\frac{x^{2}}{2}} \\
H_{2}(x)=x^{2}-1
\end{gathered}
$$

- Orden 3:

$$
\begin{gathered}
H_{3}(x)=(-1)^{3} \frac{d^{3}}{d x^{3}}\left(e^{-\frac{x^{2}}{2}}\right) e^{\frac{x^{2}}{2}} \\
H_{3}(x)=(-1)\left(-x^{3}+3 x\right) e^{-\frac{x^{2}}{2}} e^{\frac{x^{2}}{2}} \\
H_{3}(x)=x^{3}-3 x
\end{gathered}
$$


- Orden 4:

$$
\begin{gathered}
H_{4}(x)=(-1)^{4} \frac{d^{4}}{d x^{4}}\left(e^{-\frac{x^{2}}{2}}\right) e^{\frac{x^{2}}{2}} \\
H_{4}(x)=(1)\left(x^{4}-6 x^{2}+3\right) e^{-\frac{x^{2}}{2}} e^{\frac{x^{2}}{2}} \\
H_{4}(x)=x^{4}-6 x^{2}+3
\end{gathered}
$$

- Orden 5:

$$
\begin{gathered}
H_{5}(x)=(-1)^{5} \frac{d^{5}}{d x^{5}}\left(e^{-\frac{x^{2}}{2}}\right) e^{\frac{x^{2}}{2}} \\
H_{5}(x)=(-1)\left(-x^{5}+10 x^{3}+15 x\right) e^{-\frac{x^{2}}{2}} e^{\frac{x^{2}}{2}} \\
H_{5}(x)=x^{5}-10 x^{3}+15 x
\end{gathered}
$$

- Orden 6:

$$
\begin{gathered}
H_{6}(x)=(-1)^{6} \frac{d^{6}}{d x^{6}}\left(e^{-\frac{x^{2}}{2}}\right) e^{\frac{x^{2}}{2}} \\
H_{6}(x)=(-1)\left(-x^{6}+15 x^{4}+45 x^{2}-15\right) e^{-\frac{x^{2}}{2}} e^{\frac{x^{2}}{2}} \\
H_{6}(x)=x^{6}-15 x^{4}+45 x^{2}-15
\end{gathered}
$$

Se realiza el cálculo de los primeros seis polinomios, porque hasta este orden se obtienen las expresiones que convergen en función de la demostración que se realiza en la siguiente sección, cuando se reescribe la función característica de $S_{n}$. 


\subsubsection{La expansión de Edgeworth}

Una vez calculados los primeros seis polinomios de Hermite, y establecida su relación con $\varnothing$, se puede reescribir la función característica de $S_{n}$ :

$$
\begin{aligned}
g_{S_{n}}(x) & =\frac{1}{2 \pi} \int_{-\infty}^{\infty} e^{-i t x} e^{-\frac{t^{2}}{2}} d t+\frac{E\left[X^{3}\right]}{6 \sqrt{n}} \frac{1}{2 \pi} \int_{-\infty}^{\infty} e^{-i t x} e^{-\frac{t^{2}}{2}}(i t)^{3} d t \\
& +\frac{\left(E\left[X^{4}\right]-3\right)}{24 n} \frac{1}{2 \pi} \int_{-\infty}^{\infty} e^{-i t x} e^{-\frac{t^{2}}{2}}(i t)^{4} d t \\
& +\frac{E\left[X^{3}\right]^{2}}{72 n} \frac{1}{2 \pi} \int_{-\infty}^{\infty} e^{-i t x} e^{-\frac{t^{2}}{2}}(i t)^{6} d t
\end{aligned}
$$

para expresarla de la siguiente forma:

$$
\begin{gathered}
g_{S_{n}}(x)=\emptyset(x)+\frac{E\left[X^{3}\right]}{6 \sqrt{n}} H_{3}(x) \emptyset(x)+\frac{\left(E\left[X^{4}\right]-3\right)}{24 n} H_{4}(x) \emptyset(x) \\
+\frac{E\left[X^{3}\right]^{2}}{72 n} H_{6}(x) \emptyset(x) \\
=\emptyset(x)\left(1+\frac{E\left[X^{3}\right]}{6 \sqrt{n}} H_{3}(x)+\frac{\left(E\left[X^{4}\right]-3\right)}{24 n} H_{4}(x)+\frac{E\left[X^{3}\right]^{2}}{72 n} H_{6}(x)\right) \\
=\varnothing(x)\left(1+\frac{E\left[X^{3}\right]}{6 \sqrt{n}}\left(x^{3}-3 x\right)+\frac{\left(E\left[X^{4}\right]-3\right)}{24 n}\left(x^{4}-6 x^{2}+3\right)\right. \\
\left.+\frac{E\left[X^{3}\right]^{2}}{72 n}\left(x^{6}-15 x^{4}+45 x^{2}-15\right)\right)
\end{gathered}
$$


Los elementos $E\left[X^{3}\right]$ y $E\left[X^{4}\right]$ son las cumulantes que representan la asimetría y la curtosis, respectivamente. El término de orden $\frac{1}{\sqrt{n}}$ corrige la aproximación normal básica para el efecto principal de la asimetría, mientras que el término de orden $\frac{1}{n}$ corrige la aproximación normal básica para el efecto principal de la curtosis y efecto secundario de la asimetría. Por tanto, $g_{S_{n}}(x)$ se puede expresar de la siguiente manera:

$$
\begin{aligned}
g_{S_{n}}(x) & =\emptyset(x)\left(1+\frac{\xi}{6 \sqrt{n}}\left(x^{3}-3 x\right)+\frac{(\gamma-3)}{24 n}\left(x^{4}-6 x^{2}+3\right)\right. \\
& \left.+\frac{\xi^{2}}{72 n}\left(x^{6}-15 x^{4}+45 x^{2}-15\right)\right)
\end{aligned}
$$

\subsubsection{Valoración de opciones con la expansión de Edgeworth}

Una vez obtenida la forma analítica de la expansión de Edgeworth, su aplicación se ajustará a la valoración de opciones financieras.

Se sabe que la distribución de los rendimientos de los activos en los mercados no es gaussiana, presenta colas pesadas y asimetría (Milanesi, 2014). Por tal motivo, estos retornos se modelarán con la aproximación de Edgeworth. La ventaja de usar la aproximación de Edgeworth es que tiene una buena forma analítica y es posible obtener una forma cerrada para conseguir el precio justo de una opción sobre ese activo (Balieiro y Rosenfeld, 2004).

Recapitulando, una opción es un contrato que le da a su comprador, a cambio del pago de una prima, el derecho - pero no la obligación- de comprar o vender un activo financiero a un determinado precio (strike o precio de ejercicio) durante un periodo determinado de tiempo o en una fecha determinada (Madaula, 2016).

Si la opción es de compra el valor de la prima está dado por:

$$
C_{T}=\operatorname{Max}\left(S_{T}-K, 0\right)
$$

Uno de los supuestos básicos del modelo es que los retornos del activo subyacente siguen un movimiento geométrico browniano: 


$$
\frac{d S}{S}=\mu d t+\sigma d W
$$

A partir de esto, se define que el precio de la opción está determinado por la ecuación diferencial parcial estocástica de Black-Scholes:

$$
\frac{\partial C}{\partial t}+\frac{1}{2} \sigma^{2} S^{2} \frac{\partial^{2} C}{\partial^{2} S}+r S \frac{\partial C}{\partial S}-r C=0
$$

Una forma alternativa de encontrar el precio de una opción es escribir la solución de la ecuación de Black-Scholes como el valor esperado del precio final al vencimiento descontado por la tasa de interés libre de riesgo:

$$
C_{0}=e^{-r T} \int_{-\infty}^{\infty} d x P(x, t) C_{T}
$$

Resolviendo la ecuación diferencial o el valor esperado, se obtiene la fórmula del precio de la opción:

$$
C_{0}=S_{0} N\left(d_{1}\right)-K e^{-r T} N\left(d_{2}\right)
$$

A partir de esto, se debe encontrar la medida riesgo neutral para aplicarla al valor esperado. En la medida libre de riesgo, el precio promedio del activo debe obedecer lo siguiente:

$$
S_{T} \int_{-\infty}^{\infty} d x g(x) S_{0} e^{\left(\mu-\sigma^{2} / 2\right) T+\sigma \sqrt{T x}}=S_{0} e^{r T}
$$

A partir de los trabajos de Baliero Filho y Rosenfeld (2004), se deriva el modelo con base en la tasa de crecimiento del activo definida como:

$$
\mu T=r T-\log \left(1+\frac{k-3}{24}(\sigma \sqrt{T})^{3}+\frac{\xi^{2}}{72}(\sigma \sqrt{T})^{6}\right)
$$


Nótese que $\mu=r$ cuando $\xi=0$ y $k=3$, es decir, se obtiene una distribución gaussiana.

Así las cosas, se puede obtener el precio de la opción de la siguiente forma:

$$
C_{0}^{E d g e .}=e^{-r T} \int_{-\infty}^{\infty} d x g(x) \operatorname{Max}\left(S_{0} e^{\left(\mu-\sigma^{2} / 2\right) T+\sigma \sqrt{T} x}-K, 0\right)
$$

Transformando la anterior integral en una solución cerrada para calcular el precio de la opción (Baliero Filho y Rosenfeld, 2004), para el caso de la expansión de Edgeworth, se obtiene la siguiente ecuación:

$$
\begin{aligned}
C_{0}^{E d g e .} & =C_{0}^{B S}+\left(\frac { e ^ { \mu - r T - x _ { m } ^ { 2 } / 2 + \sigma \sqrt { T } x _ { m } } } { 7 2 \sqrt { 2 \pi } } S \left((\sigma \sqrt{T})^{5} \xi^{2}+(\sigma \sqrt{T})^{4} \xi^{2} x_{m}\right.\right. \\
& +(\sigma \sqrt{T})^{3}\left(3(k-3) \xi^{2}\left(x_{m}^{2}-1\right)\right. \\
& +(\sigma \sqrt{T})^{2}\left(12 \xi-3(k-3) x_{m}+\xi^{2} x_{m}\left(x_{m}^{2}-1\right)\right) \\
& \left.\left.\left.+\xi^{2}\left(x_{m}^{4}-6 x_{m}^{2}+3\right)\right)\right)\right) \\
& +\left(\frac{e^{\mu-r T-x_{m}^{2} / 2}}{72 \sqrt{2 \pi}}\left(e^{\mu+\sigma \sqrt{T} x_{m}} S-K\right)\left(3(k-3) x_{m}\left(x_{m}^{2}-3\right)\right)\right. \\
& \left.\left.+12 \xi\left(x_{m}^{2}-1\right)+\xi^{2} x_{m}\left(x_{m}^{4}-10 x_{m}^{2}+15\right)\right)\right) \\
& +\left(\frac { e ^ { \mu - r T - \sigma ^ { 2 } T / 2 } } { 7 2 } S N ( d _ { 1 } ) \left((\sigma \sqrt{T})^{4} 3(k-3)+(\sigma \sqrt{T})^{6} \xi^{2}\right.\right. \\
& \left.\left.\left.+12(\sigma \sqrt{T})^{3} \xi\right)\right)\right)
\end{aligned}
$$

Donde $x_{m}=\frac{\left(\log \left(\frac{K}{S_{0}}\right)-\left(\mu-\frac{\sigma^{2}}{2}\right) T\right)}{\sigma \sqrt{T}}$ es el valor mínimo que la variable aleatoria puede tener para un integrando distinto de cero. Nótese que se obtiene la fórmula Black-Scholes cuando $\xi=0$ y $k=3$. 


\section{Caso de aplicación: valoración de una opción call sobre las acciones del Grupo Bancolombia - Bcolombia}

Se calculará una opción de compra europea que no paga dividendos sobre el precio de cierre diario de las acciones de Bancolombia negociadas en la Bolsa de Valores de Colombia para el periodo comprendido entre el 1 de febrero de 2016 y el 28 de febrero de 2020 , se tomó esta serie de datos ${ }^{4}$ para el periodo mencionado, que resume el comportamiento reciente de estas acciones en el sector financiero colombiano.

Con el objetivo de revisar los impactos derivados de la inclusión de los momentos de orden superior, en esta sección se implementarán los modelos binomiales presentados en la metodología, con el correspondiente ajuste por la expansión de Edgeworth.

El primer paso consiste en comprobar que la serie de datos es univariadamente normal. La tabla 1 muestra los coeficientes de asimetría y curtosis, los cuales indican que la distribución de las acciones de Bancolombia tiene un leve grado de sesgo a la izquierda y es leptocúrtica. Adicionalmente, la prueba de normalidad realizada a través del estadístico Anderson-Darling indica que la variable de estudio no sigue una distribución normal, con un nivel de significancia de $5 \%$.

Tabla 1: Estadísticas descriptivas

\begin{tabular}{|l|c|}
\hline $\mathrm{N}$ & 996 \\
\hline Media & 0,00054249 \\
\hline Desviación estándar & 0,014480146 \\
\hline Asimetría & $-0,19585491$ \\
\hline Curtosis & 1,95161982 \\
\hline Prueba Anderson-Darling & 6,7357 \\
\hline P-Valor & $5,0000 \mathrm{e}-04$ \\
\hline
\end{tabular}

Fuente: elaboración propia.

4 Las series de precios para estimar los rendimientos fueron obtenidas de la Bolsa de Valores de Colombia (BVC, s. f.). 
Gráficamente se puede evidenciar lo mencionado anteriormente en el histograma de rendimientos (figura 1). La presencia de estas características en una distribución empírica y su tratamiento dependen, en buena medida, de la naturaleza de los datos financieros, sobre todo porque las condiciones económicas particulares de un mercado pueden producir comportamientos irregulares y permanentes en las variables financieras.

El supuesto de normalidad que se asume en gran medida para explicar los eventos financieros es también relevante para analizar el comportamiento que tiene un activo financiero en situaciones en las cuales una economía presenta momentos de crecimiento económico, así como también, durante periodos de contracción de la economía.

En el caso colombiano podemos observar ambos escenarios, un primer caso en el cual en 1998, el país enfrentó una crisis financiera y económica de grandes proporciones que trajo severas consecuencias en materia de crecimiento económico, lo que llevó a una continua intervención del Gobierno nacional con el fin de superarla.

Figura 1: Histograma de rendimientos diarios de la acción BCOLOMBIA

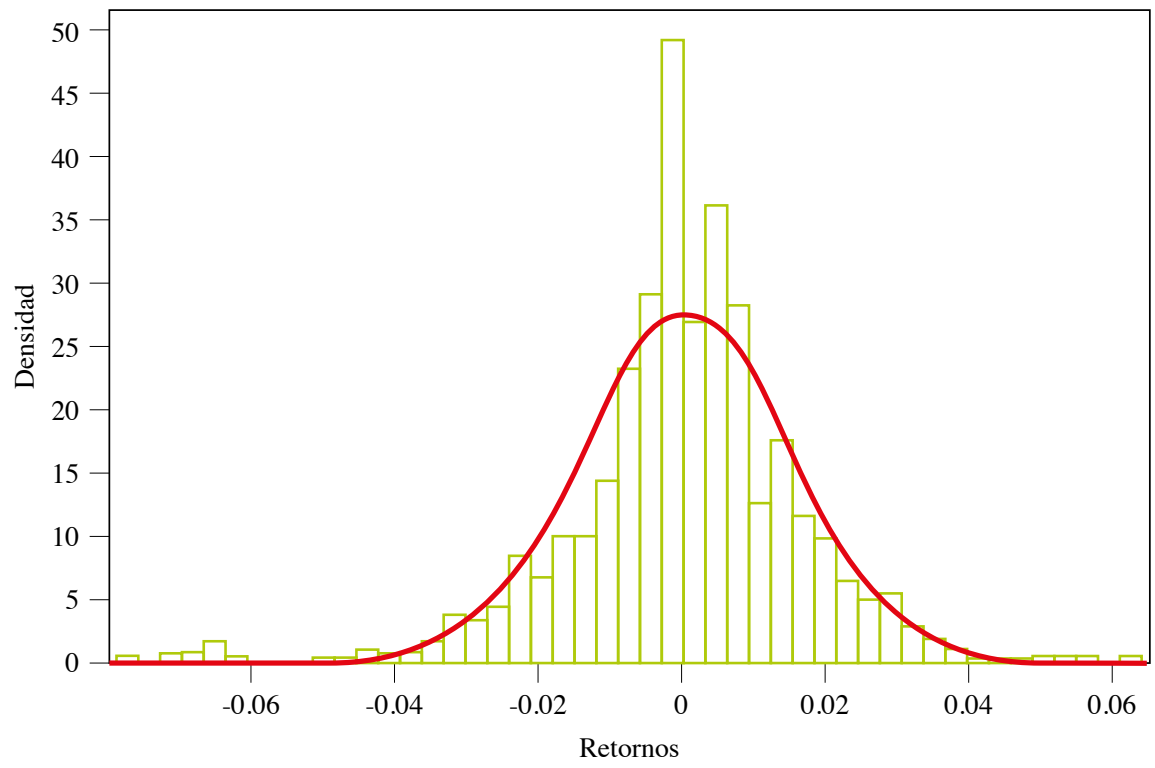

Fuente: elaboración propia. 
Figura 2: Diagrama de probabilidad normal (Q-Q plot) de la acción BCOLOMBIA

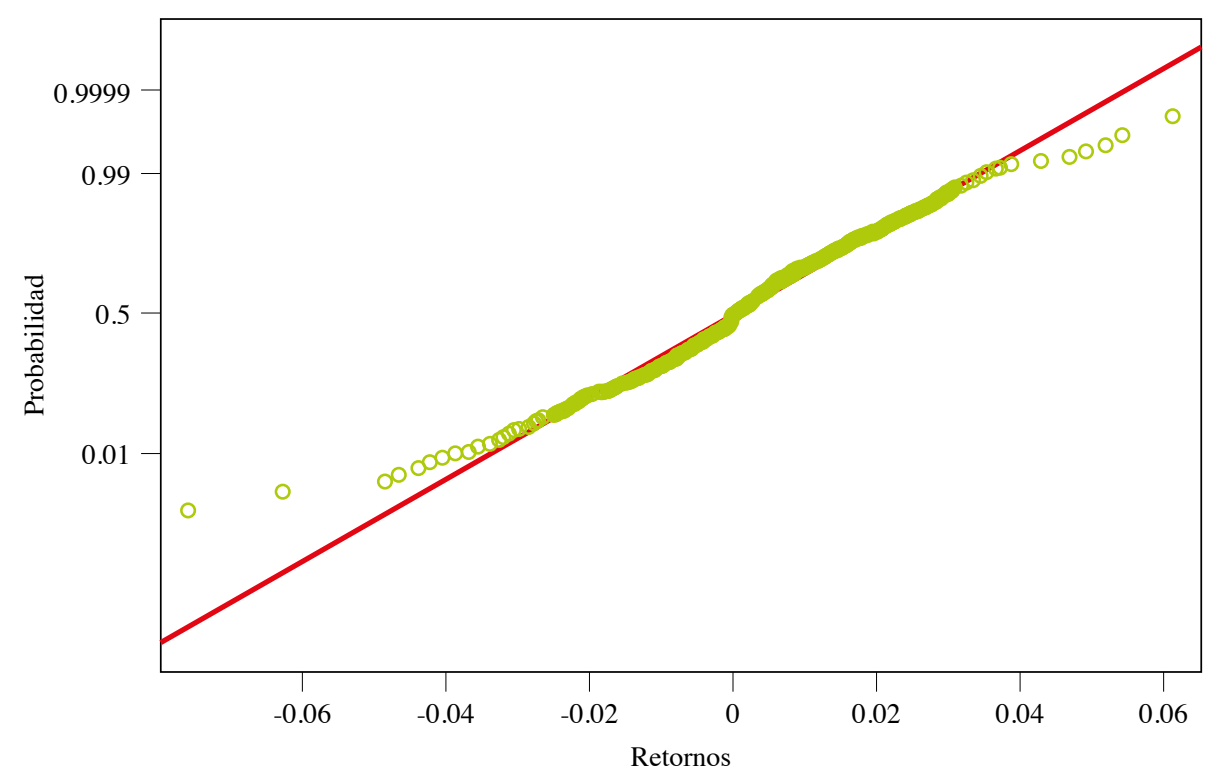

Fuente: elaboración propia.

Si se analiza el comportamiento particular de una serie financiera, en este caso, la serie de acciones de Bancolombia para el periodo 1998-2001 -periodo en el cual se dio una fase de contracción del sector real y financiero en el país-, se puede observar (figura 3) la distribución de los rendimientos de esta serie, donde se evidencia un fuerte sesgo negativo y leptocurtosis que muestra la ausencia de normalidad.

El siguiente escenario muestra cómo la economía colombiana obtiene un buen comportamiento desde el año 2003, en el cual se presenta un ciclo expansivo que se mantiene y acelera en los años siguientes hasta 2007, donde se observa uno de los mayores crecimientos registrados en la historia económica del país, dicho crecimiento permitió un incremento sostenido del PIB, logro de las metas de inflación (excepto 2007) y altas tasas de inversión; por lo anterior, a este periodo se le puede considerar de gran auge para el país.

En la figura 4 se puede evidenciar el comportamiento de la serie de acciones de Bancolombia para el periodo 2003-2007, donde se observa un sutil sesgo a la derecha y exceso de leptocurtosis que muestra la ausencia de normalidad. 
Figura 3: Histograma de rendimientos diarios de la acción BCOLOMBIA, periodo 1998-2001

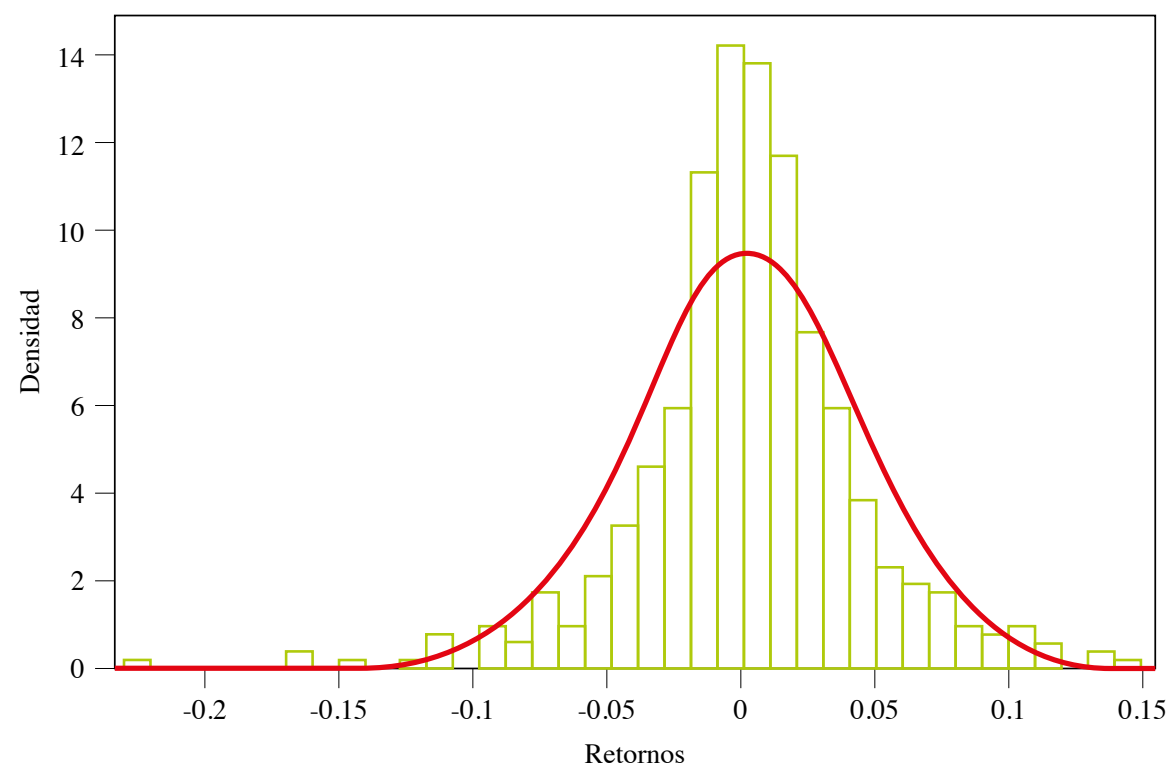

Fuente: elaboración propia.

Figura 4: Histograma de rendimientos diarios de la acción BCOLOMBIA, periodo 2003-2007

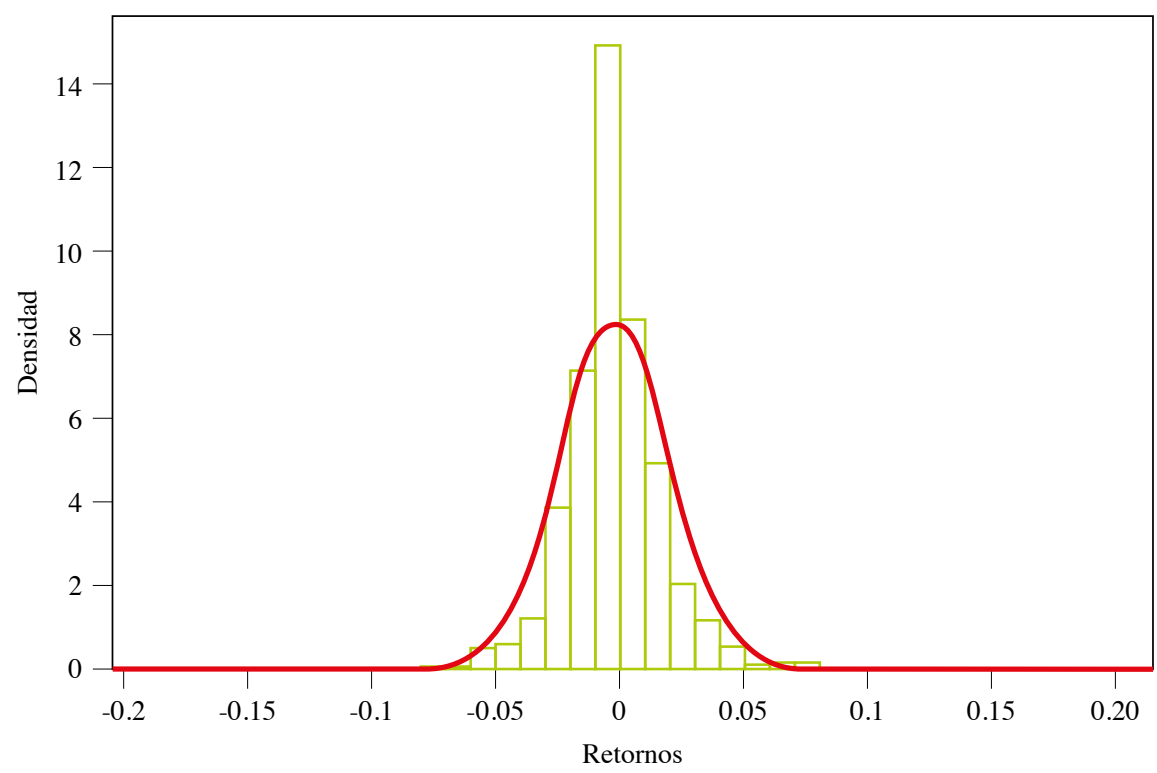

Fuente: elaboración propia. 
Una vez verificado que la serie de datos de las acciones de Bancolombia no cumple con el criterio de normalidad, se procede a calcular el valor de la opción financiera bajo los modelos binomiales, ajustándolos por la extensión aplicada por la expansión de Edgeworth.

A continuación, se presenta la información básica requerida para hacer la valoración a partir de la serie de datos de las acciones BCOLOMBIA del periodo comprendido entre el 1 de febrero de 2016 y el 28 de febrero de 2020 (tabla 2).

Tabla 2: Variables principales del modelo de valoración de opciones

\begin{tabular}{|c|l|c|}
\hline Variable & \multicolumn{1}{|c|}{ Descripción/Observación } & Valor \\
\hline$S$ & Precio de cierre de la acción al 28-02-2020 & 39.000 \\
\hline$K$ & Precio de ejercicio “At the money” & 39.000 \\
\hline$\sigma$ & Volatilidad histórica & $22,99 \%$ \\
\hline$r$ & Tasa libre de riesgo / TES en pesos con vencimiento un año & $2,97 \%$ \\
\hline$\xi$ & Asimetría & $-0,20$ \\
\hline$k$ & Curtosis & 1,95 \\
\hline$N$ & Número de pasos $(1,5,50,500,1000)$ & \\
\hline$T$ & Vencimiento & 1 año \\
\hline
\end{tabular}

Fuente: elaboración propia.

A partir de esta información, se calculó para cada uno de los modelos binomiales el precio de la opción call europea sin dividendos, así como el precio de la opción call europea sin dividendos bajo la expansión de Edgeworth, valores que se contrastaron al vencimiento con el precio obtenido mediante la metodología propuesta por Black-Scholes.

Los resultados obtenidos del proceso de valoración son los siguientes: 


\begin{tabular}{|c|c|c|c|c|c|c|}
\hline \multirow{3}{*}{ 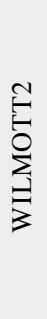 } & 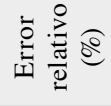 & $\stackrel{?}{\varrho}$ & $\tilde{i}$ & oे & $\begin{array}{l}n \\
0 \\
1\end{array}$ & $\begin{array}{l}n \\
0 \\
0\end{array}$ \\
\hline & 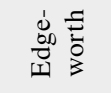 & $\hat{n}$ & $\begin{array}{l}\stackrel{0}{0} \\
\text { †े }\end{array}$ & 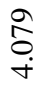 & $\begin{array}{l}\stackrel{2}{\partial} \\
\stackrel{+}{f}\end{array}$ & $\begin{array}{l}\mathfrak{n} \\
\stackrel{0}{+} \\
\dot{\gamma}\end{array}$ \\
\hline & 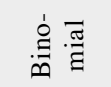 & $\underset{⿱ ㇒}{\Xi}$ & $\stackrel{m}{m}$ & 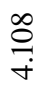 & $\underset{f}{\Xi}$ & $\begin{array}{l}\Xi \\
\exists\end{array}$ \\
\hline \multirow{3}{*}{ 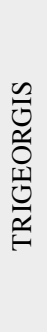 } & 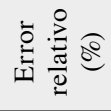 & $\nabla_{0}^{+}$ & $\stackrel{0}{=}$ & $\Rightarrow$ & $\begin{array}{l}0 \\
0 \\
i\end{array}$ & $\begin{array}{l}0 \\
0 \\
0\end{array}$ \\
\hline & 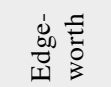 & 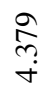 & $\underset{+}{\stackrel{\infty}{二}}$ & $\begin{array}{l}\stackrel{P}{S} \\
\dot{f}\end{array}$ & $\hat{g}$ & $\begin{array}{l}\stackrel{+}{\circ} \\
\stackrel{+}{+}\end{array}$ \\
\hline & 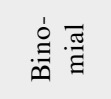 & 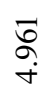 & $\begin{array}{l}\infty \\
\infty \\
\stackrel{+}{+}\end{array}$ & $\begin{array}{l}\stackrel{\partial}{\partial} \\
\dot{+}\end{array}$ & $\stackrel{\curvearrowleft}{=}$ & $\stackrel{0}{=}$ \\
\hline \multirow{3}{*}{ 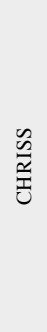 } & 站产 & $\vartheta^{\infty}$ & $\stackrel{0}{=}$ & $\stackrel{\circ}{i}$ & $\begin{array}{l}0 \\
0 \\
i\end{array}$ & $\begin{array}{l}n \\
0 \\
0\end{array}$ \\
\hline & 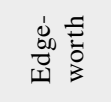 & $\hat{\text { ले }}$ & $\underset{+}{\stackrel{+}{+}}$ & $\begin{array}{l}\hat{S} \\
\text { ○ } \\
\dot{+}\end{array}$ & $\begin{array}{l}+ \\
\stackrel{+}{+}\end{array}$ & $\begin{array}{l}\mathfrak{n} \\
\stackrel{a}{+} \\
\dot{\gamma}\end{array}$ \\
\hline & $\stackrel{\dot{b}}{\stackrel{\Xi}{\Xi} \cdot \bar{\Xi}}$ & \begin{tabular}{l}
$\stackrel{0}{0}$ \\
\multirow{r}{*}{}
\end{tabular} & 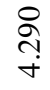 & 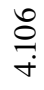 & $\underset{f}{\Xi}$ & $\stackrel{\Xi}{\ni}$ \\
\hline \multirow{3}{*}{ 总 } & 矛旀 & $\overbrace{0}^{\infty}$ & $\stackrel{0}{=}$ & $\stackrel{\circ}{\rightarrow}$ & $\begin{array}{l}0 \\
0 \\
i\end{array}$ & $\begin{array}{l}n \\
0 \\
0\end{array}$ \\
\hline & 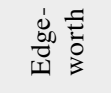 & $\hat{\text { mे }}$ & $\underset{+}{\stackrel{+}{+}}$ & $\stackrel{\hat{s}}{\circ}$ & $\begin{array}{l}t \\
\text { gे } \\
\dot{f}\end{array}$ & $\begin{array}{l}n \\
o \\
\dot{\sigma}\end{array}$ \\
\hline & 官 & \begin{tabular}{l}
$\stackrel{0}{\circ}$ \\
\multirow{+}{*}{}
\end{tabular} & 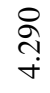 & 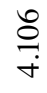 & 三 & $\stackrel{\Xi}{\ni}$ \\
\hline \multirow{3}{*}{$\stackrel{\approx}{\approx}$} & 密怘 & $\tilde{n}^{n}$ & $\stackrel{0}{=}$ & $\Rightarrow$ & $\begin{array}{l}0 \\
0 \\
i\end{array}$ & $\begin{array}{l}0 \\
0 \\
0\end{array}$ \\
\hline & 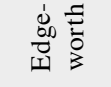 & 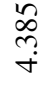 & $\underset{+}{\infty}$ & $\underset{+}{\overrightarrow{0}}$ & 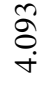 & $\begin{array}{l}+ \\
\stackrel{+}{a} \\
\dot{+}\end{array}$ \\
\hline & 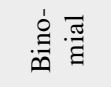 & $\begin{array}{l}\hat{b} \\
\stackrel{+}{+}\end{array}$ & 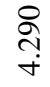 & $\begin{array}{l}\stackrel{\partial}{\circ} \\
\dot{+}\end{array}$ & $\stackrel{n}{=}$ & $\stackrel{0}{=}$ \\
\hline & z & - & $n$ & in & $\stackrel{8}{8}$ & 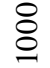 \\
\hline
\end{tabular}

ODEON, ISSN: 1794-1113, E-ISSN: 2346-2140, № 19, julio-diciembre de 2020, pp. 99-152 
Analizando los resultados obtenidos se observa que en los modelos binomiales tradicionales CRR y Trigeorgis para los tres primeros periodos, el precio de la opción va en descenso y conforme se siguen realizando más pasos en el tiempo, los valores se van acercando al resultado obtenido por el modelo B-S, hasta obtener un valor para la opción call menor cuando se realizan 1000 pasos.

En el caso de los modelos binomiales tradicionales RBJRT, Chriss y Wilmott2, los valores obtenidos para la opción call van disminuyendo en la medida en que se aumentan los pasos en cada periodo hasta igualar el valor obtenido mediante el modelo B-S, en un $N$ significativamente grande.

Aplicando la expansión de Edgeworth, los cinco modelos binomiales muestran que, en los tres primeros periodos, el precio de la opción call va disminuyendo su valor. Sin embargo, a partir del cuarto periodo el precio se eleva, no obstante, es menor al obtenido mediante el modelo B-S.

En relación con los errores de aproximación, se observa que para los cinco modelos binomiales con expansión de Edgeworth, a medida que se incrementan los pasos en el tiempo, el margen de error es menor y se va haciendo negativo toda vez que el precio de la opción call, calculado con la expansión de Edgeworth, es menor al precio calculado bajo la metodología tradicional propuesta por Black-Scholes.

Tabla 4: Precio de la opción call - Metodología Black-Scholes

\begin{tabular}{|c|c|}
\hline Variable & Valor \\
\hline$S$ & 39.000 \\
\hline$K$ & 39.000 \\
\hline$\sigma$ & $22,99 \%$ \\
\hline$r$ & $2,97 \%$ \\
\hline Plazo & 1 (año) \\
\hline Valor Opción Call B-S & 4.117 \\
\hline
\end{tabular}

Fuente: elaboración propia. 
Como se observa en la tabla 3 , a medida que la opción se acerca al vencimiento, su precio se aproxima al valor obtenido mediante la metodología clásica Black-Scholes (tabla 4) en todos los modelos aplicados, gracias a que la aproximación de la distribución binomial tiende a la distribución normal, cuando $N \rightarrow \infty$. Ahora bien, al revisar el resultado obtenido aplicando la expansión de Edgeworth, se evidencia un precio de la opción menor al obtenido bajo el modelo binomial tradicional y en la medida en la que pasa el tiempo y se acerca al vencimiento, el precio es menor al alcanzado bajo Black-Scholes.

El anterior ejercicio analítico se realizó, como se mencionó anteriormente, calculando los parámetros de la serie de acciones de Bancolombia para el periodo analizado. Sin embargo, a manera de comparación y para poder observar la sensibilidad del valor de la opción ante cambios en sus parámetros, a continuación, se replica el ejercicio cambiando algunos parámetros como el precio de ejercicio (strike), la volatilidad, la asimetría y la curtosis.

Tabla 5: Precio de la opción call - Metodología Black-Scholes (ajustando el precio de ejercicio In the money-ITM)

\begin{tabular}{|c|c|}
\hline Variable & Valor \\
\hline$S$ & 39.000 \\
\hline$K$ & 37.000 \\
\hline$\sigma$ & $22,99 \%$ \\
\hline$r$ & $2,97 \%$ \\
\hline Plazo & 1 (año) \\
\hline Valor Opción Call B-S & 5.186 \\
\hline
\end{tabular}

Fuente: elaboración propia. 


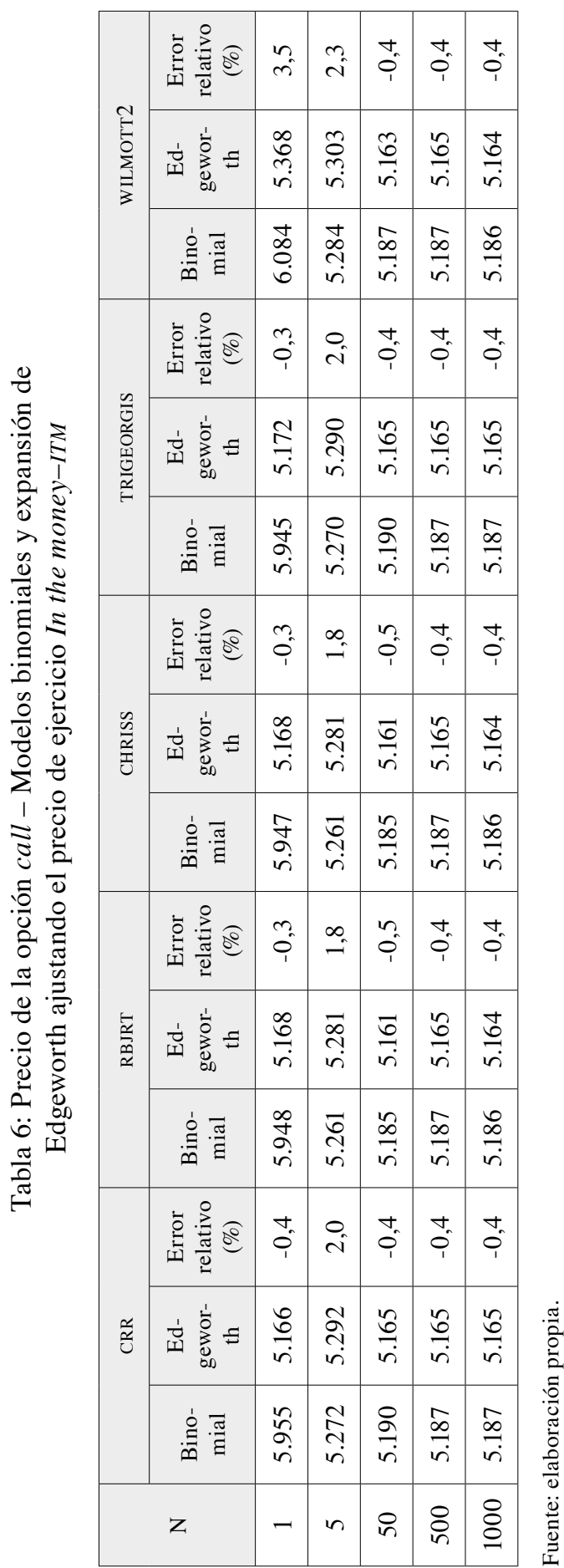


En la tabla 6 se observa que cuando el precio del activo ha superado el precio de ejercicio In the money (ITM), el valor de la opción de compra se eleva en comparación con el desarrollo anterior (tabla 3). Asimismo, se evidencia que, en un $N$ significativamente alto, el precio obtenido mediante la expansión de Edgeworth es aproximadamente $0,4 \%$ inferior al precio obtenido mediante la metodología de B-S, en los cinco modelos binomiales.

$\mathrm{Si}$ adicional al ajuste del precio de ejercicio, esta serie presentara una volatilidad del $20 \%$, el valor de la opción presentaría el siguiente comportamiento (tabla 7).

Tabla 7: Precio de la Opción Call - Metodología Black-Scholes (ajustando la volatilidad)

\begin{tabular}{|c|c|}
\hline Variable & Valor \\
\hline$S$ & 39.000 \\
\hline$K$ & 37.000 \\
\hline$\sigma$ & $20 \%$ \\
\hline$r$ & $2,97 \%$ \\
\hline Plazo & 1 (año) \\
\hline Valor Opción Call $B-S$ & 4.774 \\
\hline
\end{tabular}

Fuente: elaboración propia. 


\begin{tabular}{|c|c|c|c|c|c|c|}
\hline & 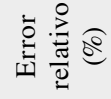 & $\hat{0}$ & $\begin{array}{l}0 \\
0\end{array}$ & $\begin{array}{l}0 \\
\dot{0}\end{array}$ & $\ddot{0}_{\dot{1}}^{+}$ & $\begin{array}{l}n \\
0 \\
0\end{array}$ \\
\hline \multirow{2}{*}{$\begin{array}{l}\mathcal{N} \\
E \\
\frac{5}{3} \\
\xi\end{array}$} & 至竞 & $\begin{array}{l}n \\
\text { N̦ } \\
n\end{array}$ & $\begin{array}{l}\vec{D} \\
\infty \\
\dot{+}\end{array}$ & $\stackrel{\substack{++}}{+}$ & 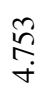 & $\stackrel{\vec{r}}{\stackrel{r}{r}}$ \\
\hline & 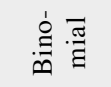 & $\begin{array}{l}\vec{\infty} \\
\stackrel{+}{+} \\
\dot{n}\end{array}$ & $\begin{array}{l}\vec{\delta} \\
\infty \\
\dot{\sigma}\end{array}$ & $\underset{f}{\stackrel{\gtrless}{f}}$ & 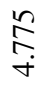 & $\stackrel{\gtrless}{\stackrel{\gtrless}{\sim}}$ \\
\hline \multirow{3}{*}{ 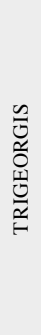 } & 站离 & o. & $\stackrel{\circ}{\rightarrow}$ & $\hat{0}_{1}$ & $\begin{array}{l}n \\
0 \\
0\end{array}$ & $\begin{array}{l}n \\
0 \\
0\end{array}$ \\
\hline & 㿝竞竞= & $\begin{array}{l}\text { ¿ } \\
\text { in }\end{array}$ & 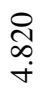 & $\underset{+}{\stackrel{F}{+}}$ & $\stackrel{\vec{n}}{\stackrel{r}{+}}$ & 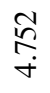 \\
\hline & 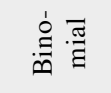 & ळे & $\begin{array}{l}\stackrel{\infty}{\infty} \\
\dot{+}\end{array}$ & $\begin{array}{l}\stackrel{8}{\circ} \\
\stackrel{+}{+}\end{array}$ & 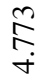 & $\underset{⿱}{\stackrel{+}{\sim}}$ \\
\hline \multirow{3}{*}{ 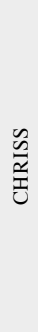 } & 竞: & $\stackrel{\sim}{\infty}$ & $\overbrace{0}^{m}$ & $\begin{array}{l}0 \\
\stackrel{0}{1}\end{array}$ & $\ddot{o}_{i}$ & $\begin{array}{l}n \\
0 \\
0\end{array}$ \\
\hline & 㿝竞 & $\frac{\vec{t}}{\frac{1}{n}}$ & $\begin{array}{l}\stackrel{\infty}{\infty} \\
\stackrel{\infty}{f}\end{array}$ & $\stackrel{\stackrel{n}{+}}{\stackrel{f}{+}}$ & 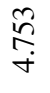 & $\stackrel{\vec{r}}{\stackrel{r}{+}}$ \\
\hline & 官 & হి & $\begin{array}{l}\stackrel{0}{\infty} \\
\stackrel{+}{f}\end{array}$ & $\begin{array}{l}\stackrel{\partial}{\circ} \\
\dot{+}\end{array}$ & 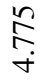 & $\underset{r}{\stackrel{n}{r}}$ \\
\hline \multirow{3}{*}{$\begin{array}{l}\underline{\underline{a}} \\
\stackrel{0}{\approx}\end{array}$} & 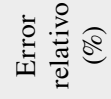 & $\stackrel{N}{\infty}$ & $3_{0}^{m}$ & $\begin{array}{l}0 \\
\dot{0}\end{array}$ & $\ddot{\sigma}_{i}^{+}$ & $\begin{array}{l}n \\
0 \\
i\end{array}$ \\
\hline & 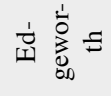 & $\frac{\vec{t}}{\operatorname{rn}}$ & $\begin{array}{l}\stackrel{\infty}{\triangleright} \\
\dot{+}\end{array}$ & 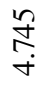 & 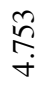 & $\stackrel{\vec{r}}{\stackrel{r}{r}}$ \\
\hline & 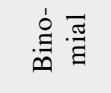 & $\begin{array}{l}\overline{\text { nे }} \\
\text { n. }\end{array}$ & 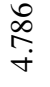 & $\begin{array}{l}\stackrel{\partial}{\stackrel{f}{*}} \\
\dot{+}\end{array}$ & 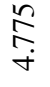 & 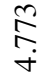 \\
\hline \multirow{3}{*}{$\stackrel{\approx}{\approx}$} & 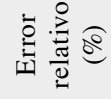 & $\vec{\infty}$ & $\Rightarrow$ & $\hat{0}_{i}$ & $\begin{array}{l}n \\
\tilde{o}_{1}\end{array}$ & $\begin{array}{l}n \\
0 \\
0\end{array}$ \\
\hline & 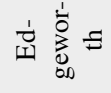 & $\frac{\wp}{\stackrel{9}{n}}$ & $\begin{array}{l}\stackrel{2}{ } \\
\infty \\
\dot{+}\end{array}$ & $\stackrel{\stackrel{Y}{+}}{\stackrel{+}{+}}$ & $\stackrel{\vec{r}}{\stackrel{r}{r}}$ & 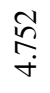 \\
\hline & 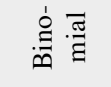 & 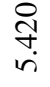 & $\begin{array}{l}\stackrel{2}{1} \\
\infty \\
+ \\
+\end{array}$ & $\begin{array}{l}\stackrel{i}{\circ} \\
\stackrel{+}{+}\end{array}$ & 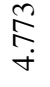 & $\underset{⿱}{\stackrel{ }{*}}$ \\
\hline & z & - & in & in & $\stackrel{8}{8}$ & $\stackrel{8}{8}$ \\
\hline
\end{tabular}


De forma similar al resultado obtenido en la tabla 6 , en este ejercicio se observa un precio para la opción call (tanto en el modelo B-S como en los modelos binomiales tradicionales y con expansión de Edgeworth) mayor al desarrollo inicial (tabla 3). Sin embargo, el valor de la prima en este ejercicio es menor que el obtenido en la tabla 6 , debido a que el efecto de reducción de la volatilidad puede suponer una disminución en el valor de la prima.

Por último, a continuación, se presenta el ejercicio asumiendo que la serie muestra una asimetría de $-0,45$ y una curtosis de 2,37. Estos valores se seleccionaron deliberadamente, pero manteniendo el comportamiento asimétrico y leptocúrtico que presenta la serie de datos.

Tabla 9: Precio de la opción call - Metodología Black-Scholes (ajustando el precio de ejercicio, la asimetría y curtosis)

\begin{tabular}{|c|c|}
\hline Variable & Valor \\
\hline$S$ & 39.000 \\
\hline$K$ & 37.000 \\
\hline$\sigma$ & $22,99 \%$ \\
\hline$r$ & $2,97 \%$ \\
\hline Plazo & $1(\mathrm{año})$ \\
\hline Valor Opción Call B-S & 5.186 \\
\hline
\end{tabular}

Fuente: elaboración propia. 


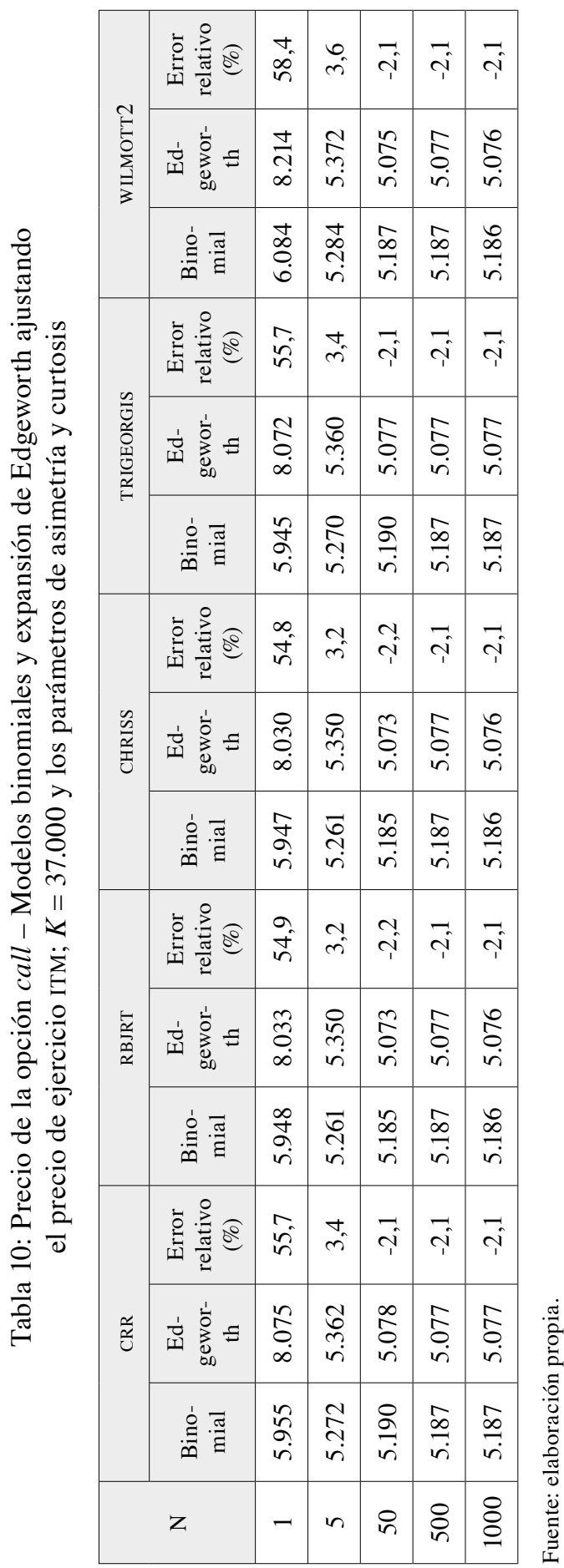


Con una asimetría negativa, se observa que en los casos ITM, la expansión de Edgeworth produce precios aproximadamente un 2,1\% más bajos que los precios ITM en B-S, en un $N$ significativamente grande.

En general, se observa que los cambios realizados a los parámetros (tanto en el precio de ejercicio, la volatilidad, la asimetría y la curtosis) para el cálculo del valor de la opción call permiten evidenciar que, en periodos cortos de tiempo, tanto los modelos binomiales tradicionales como los modelos binomiales ajustados por la expansión de Edgeworth producen valores para la opción que se alejan del valor obtenido mediante la metodología B-S. No obstante, en la medida en la que aumenta el número de pasos en el tiempo convergen rápidamente, hasta generar precios por debajo del obtenido por B-S.

Las diferencias en los precios de las opciones call entre los modelos muestran la información no capturada por el uso de la distribución gaussiana en periodos donde el comportamiento de la serie está alejado de la normalidad, como se evidencia en los valores obtenidos en asimetría y curtosis para la serie de Bancolombia. Como se mencionó, dichos parámetros reflejan aspectos susceptibles de las variables ante eventos económicos y de diversa índole.

Ahora bien, como se describió en la metodología, la expansión de Edgeworth parte del precio obtenido mediante la metodología B-S más una fracción explicada por los momentos estadísticos de orden superior. Por tanto, la diferencia en los precios evidenciados entre el modelo B-S y la obtenida mediante la aproximación es el resultado de la existencia de excesos (hacia arriba o hacia abajo) de la asimetría y la curtosis presente en la serie de datos analizada.

\section{Conclusiones}

El modelo de Black-Scholes es la metodología más aplicada en la valoración de opciones financieras. Sin embargo, como se ha mencionado en esta investigación, presenta deficiencias que se manifiestan al contrastar las predicciones obtenidas con los precios evidenciados en el mercado. Es claro que las debilidades de esta metodología son producto de los supuestos simplificadores sobre los cuales fue construida (Milanesi, 2014).

En línea con lo anterior, el objetivo de esta investigación consistió en desarrollar e implementar una técnica que involucró los momentos estadísticos de orden superior, y observar su impacto en el precio de las opciones call. Para tal fin, se empleó la expansión de Edgeworth sobre modelos binomiales, cuyos supuestos no difieren de los supuestos generalizados del modelo Black-Scholes, 
sobre todo con un $N$ muy grande, para incorporar en la estimación estas anomalías evidenciadas en los mercados financieros.

Esta técnica ajusta el método tradicional binomial de la valoración de opciones financieras al incluir momentos estadísticos de orden superior, es decir, asimetría y curtosis, con el fin de que dichas desviaciones con respecto a la distribución normal permitan identificar el comportamiento de los retornos de un activo, aspecto fundamental para la gestión del riesgo.

En la práctica, se empleó esta técnica sobre los precios de las acciones diarias del Grupo Bancolombia, contrastando sus resultados con los modelos binomiales tradicionales y frente al clásico modelo Black-Scholes. Los resultados obtenidos demuestran que incluir en el modelo de valoración aquellos aspectos que se ajustan a las expectativas del inversor y aquellas situaciones observadas en las series financieras como, por ejemplo, factores ambientales, tanto internos como externos a la actividad bursátil, le permitirán al inversionista contar con las herramientas necesarias para la toma de decisiones.

Cabe aclarar que, aunque los resultados obtenidos no son concluyentes ya que se utilizó un solo caso de aplicación, sí son consistentes con las presunciones sobre el precio de la opción, tales como: el valor teórico de la opción obtenido mediante el modelo binomial transformado está compuesto por el resultado obtenido por el método Black-Scholes más el aporte generado por los momentos estadísticos de orden superior.

Es clara la práctica generalizada de estimar las medidas financieras con base en metodologías que se fundan en el supuesto de normalidad, principalmente como resultado del Teorema del Límite Central y con la facilidad de poder aplicar los fundamentos estadísticos de la distribución normal para predecir el comportamiento de las variables financieras. Sin embargo, las distribuciones empíricas de los rendimientos de las acciones presentan ligeros sesgos negativos durante las crisis, porque los traders tienden a reaccionar más decididamente a las noticias negativas que a las positivas en situaciones de emergencia; y mayor leptocurtosis en épocas de auge y recesión porque los portafolios que incluyen rendimientos mayores son más sensibles que los demás a los cambios bruscos registrados en los precios de los activos líderes (Rydberg, 2000).

A lo largo de la literatura se han establecido supuestos frente a las causas de la existencia de asimetría y curtosis en los activos financieros; en general, se supone que su existencia es producto de cambios en la estructura de capital, eventos exógenos al mercado o por la mayor varianza de los rendimientos en algunos días de la semana (Ramírez y Chacón, 2014). 
Las condiciones propias del mercado colombiano hacen pensar que la presencia de asimetría y curtosis en las distribuciones de los rendimientos son, también, producidas por causas no necesariamente endógenas a su funcionamiento (Ferrari y González, 2007). En particular, los movimientos característicos de los precios líderes de las economías emergentes, las tasas de inflación, el tipo de cambio y las tasas de interés son comúnmente citados como fuente de variación importante de los rendimientos de las acciones preferidas por los grandes inversionistas (Abdalla y Murinde, 1997).

Así las cosas, existen diferentes razones -que se salen del ámbito de estudio del presente trabajo- que explican la existencia de momentos de alto orden en la distribución de los rendimientos de las acciones que, de una u otra manera, complican la elección del modelo que se va a implementar, por el simple hecho de las bases estadísticas sobre las cuales se estructura. Es evidente que salirse del mundo normal implica enormes riesgos, no solo se pierde la certeza estadística que regularmente se les atribuye a los pronósticos de rendimientos o a las estimaciones del riesgo de mercado, sino que ahora sus cálculos parecen reñir con la intuición económica (Abdalla y Murinde, 1997).

Según Rydberg (2000), la literatura financiera ha demostrado las mejoras predictivas de modelos como el Black-Scholes, que consideran que los logaritmos de los precios de los activos se comportan, bajo ciertas condiciones de normalidad, como una solución a la ecuación diferencial estocástica. Sin embargo, no es suficiente hacer construcciones matemáticas y estadísticas sin antes corregir los problemas que presentan los supuestos de normalidad, cuando estos problemas dependen de variables endógenas y exógenas particulares del mercado financiero, cuya solución será elegir un modelo que genere la información de mayor calidad que conduzca a obtener un precio lo más robusto posible, y que incorpore el conocimiento cualitativo del mercado. De lo contrario, el pronóstico obtenido no cumplirá con su papel de ser un instrumento de decisión financiera.

En síntesis, este trabajo desarrolló e implementó una técnica que permite valorar opciones financieras incorporando los momentos estadísticos de orden superior de los activos financieros, bajo la simplicidad que ofrece el modelo binomial y su compatibilidad de análisis con el modelo Black-Scholes. Todos los esfuerzos que se orienten a desarrollar metodologías alternativas que permitan explicar los comportamientos anormales de las variables financieras contribuirán de forma significativa al complejo campo de la teoría financiera y serán de gran utilidad para los inversionistas potenciales o reales en la administración de portafolios. 
Finalmente, entre las principales extensiones producto de la presente investigación, se plantea observar el comportamiento de una opción put al incluir la expansión de Edgeworth en los procesos de valoración binomial. También sería importante analizar los factores exógenos a las variables económicas, determinar su relación causal, el impacto sobre el activo y la forma de incluirlos en los métodos de estimación, lo cual puede ofrecer información muy útil para los procesos de valoración financiera.

\section{Referencias}

Abdalla, I. y Murinde, V. (1997). Exchange rate and stock price interactions in emerging financial markets: Evidence on India, Korea, Pakistan and the Philippines. Applied Financial Economics, 7, 25-35.

Ahmed (2006). Mir's Trinomial Expansion Theorem Based on Exponent Array Model. http://mathonline.wikidot.com/the-trinomial-theorem.

Akita, T., Jin, J. y Wakaki, H. (2010). High-dimensional Edgeworth expansion of a test statistic on independence and its error bound. Journal of Multivariate Analysis, 101(8), 1806-1813. https://doi.org/10.1016/j.jmva.2010.03.014

Arnica, G. (1993). Reflexiones sobre la validez del modelo de Black-Scholes. Revista Española de Financiación y Contabilidad, 22(77), 919-936. http://www.jstor. org/stable/42781016

Baliero Filho, R. y Rosenfeld, R. (2004). Testing option pricing with Edgeworth expansion. Physica A: Statistical Mechanis an its Application, 344, 484-490.

Basna, R. (2010). Edgeworth Expansion and Saddle Point Approximation for Discrete Data with Application to Chance Games. Digitala Vetenskapliga Arkivet.

Black, F. y Scholes, M. (1973). The pricing of options and corporate liabilities. Journal of Political Economy, 81(3).

Bock, A. (2014). Edgeworth Expansions for Binomial Trees. University of Kaiserslautern, Department of Mathematics. 
Brenn, T., Stian, I. y Anfinsen, N. (2017). A revisit of the Gram-Charlier and Edgeworth series expansions. University of Norway, Department of Physics and Technology, 1-12. https://munin.uit.no/bitstream/handle/10037/11261/article.pdf?sequence=1.

BVC (s. f.). Mercado de renta variable. https://www.bvc.com.co/pps/tibco/portalbvc/ Home/Mercados/enlinea/acciones

Cañada, A. (2015). Series de Taylor y Series de Fourier: un estudio comparativo. Universidad de Granada.

Chance, D. M. (2007). A Synthesis of binomial option pricing models for lognormally distributed assets. Louisiana State University. https://doi.org/10.2139/ssrn.969834.

Cox, J., Ross, S. y Rubinstein, M. (1979). Option pricing: A simplified approach. Journal of Financial Economics, 7(3), 229-263.

Dávalos, P. G. (2012). Análisis de Fourier y sus aplicaciones en el estudio de señales analógicas. Universidad Nacional del Sur

Del Brio, E., Ñíguez, T. y Perote, J. (2008). Multivariate Gram-Charlier Densities. MPRA Paper No. 29073 (46733), 6-25.

Drimus, G., Farkas, W., Necula, C. y Sokko, A. (2013). Closed form option pricing under generalized hermite expansions. SCIEX-NMS Fellowship, 1-15. https://doi. org/10.2139/ssrn.2349868.

Ferrari, C. y González, A. (2007). Fundamentales empresariales y económicos en la valoración de acciones: el caso de la bolsa colombiana. Cuadernos de Administración, 20(33).

Ferreira, C. A. (2015). Valuación de empresas y proyectos de base tecnológica [Tesis Doctoral]. Universidad Nacional del Sur.

Filho, R. G. B. y Rosenfeld, R. (2004). Testing option pricing with the Edgeworth expansion. Physica A: Statistical Mechanics and its Applications, 344(3-4 SPEC. ISS.), 484-490. https://doi.org/10.1016/j.physa.2004.06.018.

Hunter, D. R. (2014). Notes for a graduate-level course in asymptotics for statisticians. http://personal.psu.edu/drh20/asymp/lectures/asymp.pdf. 
Jarrow, R. y Rudd, A. (1982). Approximate option valuation for arbitrary stochastic processes. Journal of Financial Economics, 10, 347-369.

Kao, E. P. C. y Xie, W. (2017). Pricing spread options by generalized bivariate edgeworth expansion. International Journal of Financial Engineering, 04(02n03), 1750017. https://doi.org/10.1142/s2424786317500177.

Madaula, O. (2016). Superficie de volatilidad e interpolación de opciones del Ibex no cotizadas (trabajo final de máster). Facultad de Economía y Finanzas, Universitat de Barcelona.

Milanesi, G. (2011). Rejillas binomiales implícitas, asimetría y curtosis en la valuación de opciones reales y financieras. XXXI Jornadas Nacionales de Administración Financiera. Docentes de Administración Financiera. Universidad Nacional del Sur.

Milanesi, G. (2012). Árboles binomiales implícitos (IBT) y la valuación opciones financieras. Un caso de aplicación. Universidad Nacional del Sur, Departamento de Ciencias de la Administración.

Milanesi, G. (2013). Asimetría y curtosis en el modelo binomial para valorar opciones reales: caso de aplicación para empresas de base tecnológica. Estudios Gerenciales Universidad ICESI Colombia, 29(128), 368-378. https://doi.org/10.1016/j. estger.2013.09.011.

Milanesi, G. (2014). Momentos estocásticos de orden superior y la estimación de la volatilidad implícita: aplicación de la expansión de Edgeworth en el modelo Black-Scholes. Estudios Gerenciales, 30(133), 336-342. https://doi.org/10.1016/j. estger.2014.01.021.

Milanesi, G. y El Alabi, E. (2018). A Binomial Model with Edgeworth Expansion on Particular Circumstances. European Accounting and Management Review, 5(1), 69-93. https://doi.org/10.26595/eamr.2014.5.1.4.

Podolskij, M. y Yoshida, N. (2018). Edgeworth expansion for functionals of continuous diffusion processes. Annals of Applied Probability, 26(6), 3415-3455. https://doi. org/10.1214/16-AAP1179.

Qumsiyeh, M. (1990). Edgeworth expansion in regression models. Indiana University and Bethlehem University, 101, 86-101. 
Ramírez, J. y Chacón, O. (2014). El supuesto de normalidad estacionaria y los Black Swans en finanzas. Sotavento M.B.A. 24 (dic. 2014), 24-43.

Rodríguez, A. C. N. (2014). Aproximaciones uniformes para distribuciones de probabilidad de sumas de variables aleatorias positivas con colas pesadas. Universidad Autonoma de Madrid Escuela Politécnica Superior.

Rubinstein, M. (1998). Edgeworth binomial trees. Journal of Derivatives, 14, 20-27.

Rydberg, T. (2000). Realistic Statistical Modeling of Financial Data. International Statistical Review, 68 (3), 233-258.

Serbachi (s. f.). Serie de Taylor y Maclaurin. https://fisicauv.jimdofree.com/app/download/8922881368/Serie+de+Taylor.pdf?t=1505700219.

Simonato, J. G. (2009). Johnson binomial trees. Quantitative Finance, 11(8), 1165-1176. https://doi.org/10.1080/14697680902950821.

Taboga, M. (2020). Characteristic function. https://www.statlect.com/fundamentalsof-probability/characteristic-function 